\title{
Efficient Post-Macrocyclization Functionalizations of Oxacalix[2]arene[2]pyrimidines
}

Wim Van Rossom, ${ }^{\dagger}$ Wouter Maes,${ }^{\dagger}$ Lingam Kishore,${ }^{\dagger}$ Margriet Ovaere,${ }^{\ddagger}$ Luc Van Meervelt, ${ }^{\ddagger}$ and Wim Dehaen ${ }^{\star}{ }^{\dagger}$

\footnotetext{
${ }^{\dagger}$ Molecular Design and Synthesis, Department of Chemistry, Katholieke Universiteit Leuven, Celestijnenlaan 200F, 3001 Leuven, Belgium

${ }^{\ddagger}$ Biomolecular Architecture, Department of Chemistry, Katholieke Universiteit Leuven, Celestijnenlaan 200F, 3001 Leuven, Belgium

wim.dehaen@chem.kuleuven.be; Fax: +3216327990; Tel.: +3216327439
}

\section{Table of contents}

1. General experimental methods $\quad$ S2

2. Experimental and characterization data $\quad \mathrm{S} 2$

3. ${ }^{1} \mathrm{H}$ and ${ }^{13} \mathrm{C}$ NMR spectra for oxacalix[2] arene[2]pyrimidines 3a-d, 4, 5, 7a-1, and $\mathbf{9} \quad \mathrm{S} 7$

4. X-ray crystallographic structure and data for oxacalix[2]arene[2]pyrimidine 7k $\quad$ S26 


\section{General experimental methods}

NMR spectra were acquired on commercial instruments (Bruker Avance $300 \mathrm{MHz}$, Bruker AMX 400 $\mathrm{MHz}$ or Bruker Avance $\mathrm{II}^{+} 600 \mathrm{MHz}$ ) and chemical shifts $(\delta)$ are reported in parts per million (ppm) referenced to tetramethylsilane or the internal (NMR-)solvent signals. ${ }^{1}$ Detailed ${ }^{13} \mathrm{C}$ NMR peak assignments were obtained by careful analysis of DEPT, HMQC and HMBC NMR spectra. Mass spectra were run using a HP5989A apparatus (CI and EI, $70 \mathrm{eV}$ ionisation energy) with Apollo 300 data system or a Thermo Finnigan LCQ Advantage apparatus (APCI/ESI). Exact mass measurements were acquired on a Kratos MS50TC instrument (performed in the EI mode at a resolution of 10000) or a Fourier Transform Ion-Cyclotron Resonance (FTICR) mass spectrometer (positive ESI mode, resolution 95000). MALDI-TOF mass spectra were obtained with dithranol (in $\mathrm{CHCl}_{3}$ ) as a matrix. Melting points (not corrected) were determined using a Reichert Thermovar apparatus. For column chromatography 70-230 mesh silica 60 (E. M. Merck) was used as the stationary phase. MPLC flash chromatography was performed using a Büchi Sepacore ${ }^{\mathrm{TM}}$ Flash apparatus. Chemicals received from commercial sources were used without further purification. $\mathrm{K}_{2} \mathrm{CO}_{3}$ (anhyd, granulated) was finely ground (with mortar and pestle) prior to use. Extra dry DMSO (over molecular sieves, $\mathrm{H}_{2} \mathrm{O} \leq 0.005 \%$ ) was purchased from Fluka. Other solvents (DMF, $\mathrm{CH}_{3} \mathrm{CN}$, THF) were used as received from commercial sources and not explicitely dried prior to use $\left(\mathrm{H}_{2} \mathrm{O} \leq 0.1 \%\right)$.

\section{Experimental and characterization data}

\section{Liebeskind-Srogl cross-coupling}

General procedure 1: To a stirred mixture of 5,17-bis(methylsulfanyl)oxacalix[2]arene[2]pyrimidine $1(100 \mathrm{mg}, 0.20 \mathrm{mmol})$ and $\mathrm{Pd}\left(\mathrm{PPh}_{3}\right)_{4}(12 \mathrm{mg}, 5 \mathrm{~mol} \%)$ in THF $(5 \mathrm{~mL})$ under $\mathrm{Ar}$, the respective arylboronic acid 2a-d (0.61 mmol, 3 equiv) and CuTC (139 mg, $0.71 \mathrm{mmol}, 3.5$ equiv) were added, and the resulting mixture was heated at reflux for $24 \mathrm{~h}$ under an Ar atmosphere. After cooling to rt, THF was evaporated in vacuum and the residue was dissolved in $\mathrm{CH}_{2} \mathrm{Cl}_{2}$, washed with distilled water and the organic fraction was dried over $\mathrm{MgSO}_{4}$ and filtered. The respective oxacalix[4]arenes 3a-d were purified by column chromatography (silica) and obtained as white solids.

4,6,16,18-tetraaza-11,23-dimethyl-5,17-bis(4-tert-butylphenyl)-2,8,14,20-tetraoxacalix[4]arene (3a): 4-tert-Butylphenylboronic acid (2a) $(108 \mathrm{mg}, 0.61 \mathrm{mmol})$; Eluent $\mathrm{CH}_{2} \mathrm{Cl}_{2}$-heptane 9-1; Yield 68\% (93 mg); mp 224-225 ${ }^{\circ} \mathrm{C}$; MS (APCI+) m/z $665.4\left[\mathrm{MH}^{+}\right]$; HRMS (ESI) calcd for $\mathrm{C}_{42} \mathrm{H}_{40} \mathrm{~N}_{4} \mathrm{O}_{4}$ : 664.3050; found: $m / z 665.3105\left[\mathrm{MH}^{+}\right] ;{ }^{1} \mathrm{H}$ NMR $\left(300 \mathrm{MHz}, \mathrm{CDCl}_{3}\right) \delta 8.41(\mathrm{~d}, J=9.1 \mathrm{~Hz}, 4 \mathrm{H} ; m-t$-Bu$\mathrm{Ph}$ ), 7.50 (d, $J=8.2 \mathrm{~Hz}, 4 \mathrm{H}$; o-t-Bu-Ph), 6.94 (d, $J=1.8 \mathrm{~Hz}, 4 \mathrm{H}$; 4,6-orc), 6.70 (s, 2H; 2-orc), 5.33 (s, 2H; 5-pyr), 2.39 (s, 6H; $\left.\mathrm{CH}_{3}\right), 1.37\left(\mathrm{~s}, 18 \mathrm{H} ; t\right.$-Bu); ${ }^{13} \mathrm{C} \mathrm{NMR}\left(75 \mathrm{MHz}, \mathrm{CDCl}_{3}\right) \delta 173.0(\mathrm{C} ; 4,6-$ pyr $)$, 166.5 (C; 2-pyr), 155.4 (C; i-t-Bu-Ph), 153.0 (C; 1,3-orc), 143.8 (C; 5-orc), 133.2 (C; p-t-Bu-Ph), 128.6 $(\mathrm{CH} ; \mathrm{Ph}), 125.6(\mathrm{CH} ; \mathrm{Ph}), 120.6(\mathrm{CH} ; 4,6$-orc), $111.8(\mathrm{CH} ; 2$-orc), $85.1(\mathrm{CH} ; 5$-pyr), $35.1(\mathrm{C} ; t$ - $\mathrm{Bu})$, $31.3\left(\mathrm{CH}_{3} ; t-\mathrm{Bu}\right), 21.6\left(\mathrm{CH}_{3} ;\right.$ orc $)$.

4,6,16,18-tetraaza-11,23-dimethyl-5,17-bis(4-methoxyphenyl)-2,8,14,20-tetraoxacalix[4]arene (3b): 4-Methoxyphenylboronic acid (2b) $\left(93 \mathrm{mg}, 0.61 \mathrm{mmol}\right.$ ); Eluent $\mathrm{CH}_{2} \mathrm{Cl}_{2}$-heptane 4-1; Yield 78\% (97 mg); mp $>325{ }^{\circ} \mathrm{C}$; MS (APCI+) m/z 613.6 [MH ${ }^{+}$; HRMS (EI) calcd for $\mathrm{C}_{36} \mathrm{H}_{28} \mathrm{~N}_{4} \mathrm{O}_{6}$ : 612.2009; found: $\mathrm{m} / \mathrm{z}$ 612.2008; ${ }^{\mathrm{H}} \mathrm{HMR}\left(400 \mathrm{MHz}, \mathrm{CDCl}_{3}\right) \delta 8.45\left(\mathrm{~d}, J=9.1 \mathrm{~Hz}, 4 \mathrm{H} ; m-\mathrm{CH}_{3} \mathrm{O}-\mathrm{Ph}\right), 6.99(\mathrm{~d}, J=$ $9.1 \mathrm{~Hz}, 4 \mathrm{H} ; o-\mathrm{CH}_{3} \mathrm{O}-\mathrm{Ph}$ ), 6.94 (d, $J=1.2 \mathrm{~Hz}, 4 \mathrm{H} ; 4,6$-orc), 6.69 (s, 2H; 2-orc), 5.28 (s, 2H; 5-pyr), 3.89 (s, 6H; $\left.\mathrm{CH}_{3}-\mathrm{O}\right), 2.40$ (s, 6H; $\mathrm{CH}_{3}$-orc); ${ }^{13} \mathrm{C} \mathrm{NMR}\left(75 \mathrm{MHz}, \mathrm{CDCl}_{3}\right) \delta 173.0(\mathrm{C} ; 4,6-\mathrm{pyr}), 166.3(\mathrm{C} ; 2-$ pyr), 162.8 (C; i-MeO-Ph), 153.1 (C; 1,3-orc), 143.8 (C; 5-orc), 130.7 (CH; m-MeO-Ph), 128.6 (C; $p$ $\mathrm{MeO}-\mathrm{Ph}), 120.6\left(\mathrm{CH} ; 4,6\right.$-orc), $114.0\left(\mathrm{CH} ;\right.$ o-MeO-Ph), 111.8 (CH; 2-orc), 84.7 (CH; 5-pyr), $55.6\left(\mathrm{CH}_{3}\right.$; $\mathrm{OMe}), 21.7\left(\mathrm{CH}_{3}\right.$; orc $)$.

4,6,16,18-tetraaza-11,23-dimethyl-5,17-bis(4-cyanophenyl)-2,8,14,20-tetraoxacalix[4]arene (3c): 4-Cyanophenylboronic acid (2c) $(90 \mathrm{mg}, 0.61 \mathrm{mmol})$; Eluent $\mathrm{CH}_{2} \mathrm{Cl}_{2}$; Yield $75 \%(92 \mathrm{mg}) ; \mathrm{mp}>325^{\circ} \mathrm{C}$; MS (APCI+) m/z 603.3 [MH ${ }^{+}$; HRMS (EI) calcd for $\mathrm{C}_{36} \mathrm{H}_{22} \mathrm{~N}_{6} \mathrm{O}_{4}: 602.1703$; found: $\mathrm{m} / \mathrm{z} 602.1701 ;{ }^{1} \mathrm{H}$ NMR (400 MHz, CDCl $\left.{ }_{3}\right) \delta 8.60(\mathrm{~d}, J=8.5 \mathrm{~Hz}, 4 \mathrm{H} ; m-\mathrm{CN}-\mathrm{Ph}), 7.79$ (d, $\left.J=8.5 \mathrm{~Hz}, 4 \mathrm{H} ; o-\mathrm{CN}-\mathrm{Ph}\right), 6.98$ $(\mathrm{d}, J=1.4 \mathrm{~Hz}, 4 \mathrm{H} ; 4,6-\mathrm{orc}), 6.69$ (s, 2H; 2-orc), 5.42 (s, 2H; 5-pyr), $2.43\left(\mathrm{~s}, 6 \mathrm{H} ; \mathrm{CH}_{3}\right) ;{ }^{13} \mathrm{C} \mathrm{NMR}(100$

\footnotetext{
${ }^{1}$ Gottlieb, H. E.; Kotlyar, V.; Nudelman, A. J. Org. Chem. 1997, 62, 7512.
} 
$\left.\mathrm{MHz}, \mathrm{CDCl}_{3}\right) \delta 173.3$ (C; 4,6-pyr), 164.7 (C; 2-pyr), 153.0 (C; 1,3-orc), 144.4 (C; 5-orc), $140.0(\mathrm{C} ; \mathrm{Ph})$, 132.5 (CH; Ph), $129.3(\mathrm{CH} ; \mathrm{Ph}), 120.9$ (CH; 4,6-orc), 118.6 (C), 115.2 (C), 111.5 (CH; 2-orc), 86.5 (CH; 5-pyr), $21.7\left(\mathrm{CH}_{3}\right)$.

4,6,16,18-tetraaza-11,23-dimethyl-5,17-bis(2-methylphenyl)-2,8,14,20-tetraoxacalix[4]arene (3d): 2-Methylphenylboronic acid (2d) $\left(83 \mathrm{mg}, 0.61 \mathrm{mmol}\right.$ ); Eluent $\mathrm{CH}_{2} \mathrm{Cl}_{2}$; Yield $76 \%$ (90 mg); mp 154-155 ${ }^{\circ} \mathrm{C}$; MS (APCI+) m/z 581.4 [MH ${ }^{+}$; HRMS (ESI) calcd for $\mathrm{C}_{36} \mathrm{H}_{28} \mathrm{~N}_{4} \mathrm{O}_{4}$ : 580.2111; found: $\mathrm{m} / \mathrm{z}$ $581.2176\left[\mathrm{MH}^{+}\right] ;{ }^{1} \mathrm{H}$ NMR $\left(300 \mathrm{MHz}, \mathrm{CDCl}_{3}\right) \delta 7.97(\mathrm{~d}, J=7.3 \mathrm{~Hz}, 2 \mathrm{H} ; \mathrm{Ph}), 7.40-7.26(\mathrm{~m}, 6 \mathrm{H} ; \mathrm{Ph})$, 6.94 (s, 4H; 4,6-orc), 6.70 (s, 2H; 2-orc), 5.36 (s, 2H; 5-pyr), 2.67 (s, 6H; $\left.\mathrm{CH}_{3}-\mathrm{Ph}\right), 2.39$ (s, 6H; $\mathrm{CH}_{3-}$ orc); ${ }^{13} \mathrm{C}$ NMR (75 MHz, $\left.\mathrm{CDCl}_{3}\right) \delta 172.6$ (C; 4,6-pyr), 169.1 (C; 2-pyr), 153.0 (C; 1,3-orc), $143.8(\mathrm{C}$; 5orc), $137.9(\mathrm{C} ; \mathrm{Ph}), 136.5(\mathrm{C} ; \mathrm{Ph}), 131.6(\mathrm{CH} ; \mathrm{Ph}), 130.9(\mathrm{CH} ; \mathrm{Ph}), 130.3(\mathrm{CH} ; \mathrm{Ph}), 126.0(\mathrm{CH} ; \mathrm{Ph})$, 120.6 (CH; 4,6-orc), 111.9 (CH; 2-orc), $84.9\left(\mathrm{CH} ; 5\right.$-pyr), $21.6\left(\mathrm{CH}_{3}\right)$.

Direct synthesis of oxacalix[4]arene 3b: 4,6-Dichloro-2-( $p$-methoxyphenyl)pyrimidine ${ }^{2}$ (200 mg, $0.78 \mathrm{mmol})$, orcinol $(97 \mathrm{mg}, 0.78 \mathrm{mmol}), \mathrm{K}_{2} \mathrm{CO}_{3}(270 \mathrm{mg}, 1.95 \mathrm{mmol})$ and $18 \mathrm{C} 6(28 \mathrm{mg}, 110 \mu \mathrm{mol})$ were combined in dry DMF $\left(10 \mathrm{~mL}\right.$ ), and the mixture was vigorously stirred at $70{ }^{\circ} \mathrm{C}$ for $36 \mathrm{~h}$ (under Ar). DMF was removed under vacuum and the residue was redissolved in $\mathrm{CH}_{2} \mathrm{Cl}_{2}(50 \mathrm{~mL})$ and washed with distilled water $(3 \times 25 \mathrm{~mL})$. The organic fraction was dried over $\mathrm{MgSO}_{4}$, filtered and the solvent was removed under vacuum. Oxacalix[4] arene $\mathbf{3 b}$ was purified by column chromatography (silica, eluent $\mathrm{CH}_{2} \mathrm{Cl}_{2}$ ) and obtained as a white solid (48 $\mathrm{mg}, 20 \%$ ).

\section{Oxidation}

4,6,16,18-tetraaza-11,23-dimethyl-5,17-bis(methylsulfonyl)-2,8,14,20-tetraoxacalix[4]arene (general procedure 2): $m$-CPBA (210 mg, $1.22 \mathrm{mmol}, 6$ equiv) and $\mathrm{MgSO}_{4}(293 \mathrm{mg}, 2.44 \mathrm{mmol})$ were mixed together in $\mathrm{CH}_{2} \mathrm{Cl}_{2}(5 \mathrm{~mL})$ and stirred for $1 \mathrm{~h}$ at rt. Subsequently, oxacalix[4]arene 1 (100 $\mathrm{mg}$, $0.20 \mathrm{mmol}$ ) was added and the mixture was stirred at $\mathrm{rt}$ for $18 \mathrm{~h}$ (under Ar). The resulting solution was filtered, diluted with $\mathrm{MeOH}(25 \mathrm{~mL})$ and $\mathrm{CH}_{2} \mathrm{Cl}_{2}$ was carefully evaporated in vacuum, resulting in the formation of a white crystalline solid, which was filtered off and washed carefully with $\mathrm{MeOH}$ to afford oxacalix[4]arene 4 as a pure white solid $(108 \mathrm{mg}, 96 \%) . \mathrm{mp}>325{ }^{\circ} \mathrm{C}$; MS (APCI+) $\mathrm{m} / \mathrm{z} 557.1\left[\mathrm{MH}^{+}\right]$; HRMS (EI) calcd for $\mathrm{C}_{24} \mathrm{H}_{20} \mathrm{~N}_{4} \mathrm{O}_{8} \mathrm{~S}_{2}: 556.0723$; found: $\mathrm{m} / \mathrm{z} 556.0726 ;{ }^{1} \mathrm{H}$ NMR $\left(400 \mathrm{MHz}, \mathrm{CD}_{2} \mathrm{Cl}_{2}\right) \delta$ $6.98\left(\mathrm{~d}, J=1.8 \mathrm{~Hz}, 4 \mathrm{H} ; 4,6\right.$-orc), 6.79 (t, $J=2.0 \mathrm{~Hz}, 2 \mathrm{H} ; 2$-orc), 5.52 (s, 2H; 5-pyr), 3.32 (s, 6H; $\mathrm{CH}_{3^{-}}$ $\left.\mathrm{SO}_{2}\right), 2.39$ (s, $6 \mathrm{H} ; \mathrm{CH}_{3}$-orc); ${ }^{13} \mathrm{C}$ NMR $\left(75 \mathrm{MHz}, \mathrm{CD}_{2} \mathrm{Cl}_{2}\right) \delta 174.0$ (C; 4,6-pyr), 167.3 (C; 2-pyr), 152.9 (C; 1,3-orc), 145.5 (C; 5-orc), 121.3 (CH; 4,6-orc), 111.2 (CH; 2-orc), 90.3 (CH; 5-pyr), $39.4\left(\mathrm{CH}_{3}\right.$; $\left.\mathrm{SO}_{2} \mathrm{Me}\right), 21.6\left(\mathrm{CH}_{3} ;\right.$ orc $)$.

4,6,16,18-tetraaza-11,23-dimethyl-5-methylsulfonyl-2,8,14,20-tetraoxacalix[4]arene (5): Synthesis according to general procedure 2: $m$-CPBA (116 mg, $0.67 \mathrm{mmol}, 3$ equiv), $\mathrm{MgSO}_{4}(162 \mathrm{mg}, 1.34$ mmol), $\mathrm{CH}_{2} \mathrm{Cl}_{2}(5 \mathrm{~mL})$, mono(methylsulfanyl)oxacalix[4] arene (100 mg, $0.22 \mathrm{mmol}$ ); Yield $93 \%$ (99 $\mathrm{mg}$ ); mp 261-262 ${ }^{\circ} \mathrm{C}$; MS (APCI+) $\mathrm{m} / \mathrm{z} 479.6\left[\mathrm{MH}^{+}\right.$]; HRMS (ESI) calcd for $\mathrm{C}_{23} \mathrm{H}_{18} \mathrm{~N}_{4} \mathrm{O}_{6} \mathrm{~S}$ : 478.0947 ; found: $\left.\mathrm{m} / \mathrm{z} 479.1011 \mathrm{CMH}^{+}\right]$; ${ }^{1} \mathrm{H}$ NMR $\left(600 \mathrm{MHz}, \mathrm{CDCl}_{3}\right) \delta 8.69$ (s, 1H; 2-pyr), 6.96 (s, 2H; 4,6-orc), 6.93 (s, $2 \mathrm{H} ; 4,6$-orc), 6.67 (s, 2H; 2-orc), $5.56\left(\mathrm{~s}, 1 \mathrm{H} ; 5\right.$-pyr), $5.38\left(\mathrm{~s}, 1 \mathrm{H} ; 5\right.$-pyr), $3.36\left(\mathrm{~s}, 3 \mathrm{H} ; \mathrm{CH}_{3}-\mathrm{SO}_{2}\right)$, 2.41 (s, $6 \mathrm{H} ; \mathrm{CH}_{3}$-orc); ${ }^{13} \mathrm{C}$ NMR (100 MHz, $\left.\mathrm{CDCl}_{3}\right) \delta 173.7$ (C; 4/6-pyr), 172.7 (C; 4/6-pyr), $167.2(\mathrm{C}$; 2-pyr-SO ${ }_{2} \mathrm{Me}$ ), 160.4 (CH; 2-pyr-H), 153.1 (C; 1/3-orc), 152.5 (C; 1/3-orc), 144.7 (C; 5-orc), 121.5 (CH; 4/6-orc), 120.5 (CH; 4/6-orc), 111.2 (CH; 2-orc), 89.8 (CH; 5-pyr), 87.7 (CH; 5-pyr), $39.1\left(\mathrm{CH}_{3}\right.$; $\left.\mathrm{SO}_{2} \mathrm{Me}\right), 21.7\left(\mathrm{CH}_{3}\right.$; orc $)$.

\section{Nucleophilic aromatic substitution}

4,6,16,18-tetraaza-11,23-dimethyl-5,17-bis(phenoxy)-2,8,14,20-tetraoxacalix[4]arene (7a) (general procedure 3): To a solution of 5,17-bis(methylsulfonyl)oxacalix[2] arene[2]pyrimidine 4 (50 $\mathrm{mg}$, $0.09 \mathrm{mmol})$ in DMF $(3 \mathrm{~mL})$, phenol $(\mathbf{6 a})(17 \mathrm{mg}, 0.18 \mathrm{mmol}, 2$ equiv $), \mathrm{K}_{2} \mathrm{CO}_{3}(31 \mathrm{mg}, 0.22 \mathrm{mmol})$ and 18-crown-6 (10 mg, $0.04 \mathrm{mmol})$ were added, and the resulting mixture was stirred at $70{ }^{\circ} \mathrm{C}$ for $24 \mathrm{~h}$ (under an Ar atmosphere). DMF was evaporated in vacuum and the resulting residue was redissolved in ethyl acetate $(50 \mathrm{~mL})$ and washed with distilled water $(3 \times 25 \mathrm{~mL})$. The organic fraction was dried over $\mathrm{MgSO}_{4}$, filtered and the solvent was evaporated in vacuum. After flash column chromatographic purification (silica, eluent heptane-ethyl acetate 7-3), oxacalix[4]arene 7a was isolated as a white solid

${ }^{2}$ Hendry, J. A.; Homer, R. F. J. Chem. Soc. 1952, 328. 
(44.5 mg, 85\%). mp 138-139 ${ }^{\circ} \mathrm{C}$; MS (APCI+) m/z $585.6\left[\mathrm{MH}^{+}\right]$; HRMS (EI) calcd for $\mathrm{C}_{34} \mathrm{H}_{24} \mathrm{~N}_{4} \mathrm{O}_{6}$ : 584.1696; found: $\mathrm{m} / \mathrm{z}$ 584.1688; ${ }^{1} \mathrm{H}$ NMR $\left(300 \mathrm{MHz}, \mathrm{CDCl}_{3}\right) \delta 7.47-7.40(\mathrm{~m}, J=8.2 \mathrm{~Hz}, 4 \mathrm{H} ; \mathrm{Ph})$, $7.30-7.21(\mathrm{~m}, 6 \mathrm{H} ; \mathrm{Ph}), 6.86$ (s, 4H; 4,6-orc), 6.62 (s, 2H; 2-orc), 5.05 (s, 2H; 5-pyr), 2.35 (s, 6H; $\left.\mathrm{CH}_{3}\right)$; ${ }^{13} \mathrm{C}$ NMR (75 MHz, $\left.\mathrm{CDCl}_{3}\right) \delta 174.6(\mathrm{C} ; 4,6$-pyr), 166.4 (C; 2-pyr), 153.1 (C; 1,3-orc), $152.7(\mathrm{C} ;$ i-PhO), 144.1 (C; 5-orc), 130.1 (CH; PhO), $126.2(\mathrm{CH} ; \mathrm{PhO}), 122.1$ (CH; PhO), 121.0 (CH; 4,6-orc), 112.1 (CH; 2-orc), $82.6\left(\mathrm{CH} ; 5\right.$-pyr), $21.9\left(\mathrm{CH}_{3}\right)$.

4,6,16,18-tetraaza-11,23-dimethyl-5,17-bis(4-tert-butylphenoxy)-2,8,14,20-tetraoxacalix[4]arene (7b): Synthesis according to general procedure 3: oxacalix[4]arene 4 (100 $\mathrm{mg}, 0.18 \mathrm{mmol})$, 4-tertbutylphenol (6b) (54 mg, $0.36 \mathrm{mmol}, 2$ equiv), $\mathrm{K}_{2} \mathrm{CO}_{3}(62 \mathrm{mg}, 0.45 \mathrm{mmol}), 18$-crown-6 (20 mg, 0.08 mmol), DMF (5 mL), $70{ }^{\circ} \mathrm{C}, 24 \mathrm{~h}$; Eluent heptane-ethyl acetate 4-1; Yield 89\% (112 mg); mp 173-174 ${ }^{\circ} \mathrm{C}$; MS (APCI+) m/z 697.7 [MH ${ }^{+}$; HRMS (EI) calcd for $\mathrm{C}_{42} \mathrm{H}_{40} \mathrm{~N}_{4} \mathrm{O}_{6}: 696.2948$; found: $\mathrm{m} / \mathrm{z}$ 696.3007; ${ }^{1} \mathrm{H}$ NMR $\left(400 \mathrm{MHz}, \mathrm{CDCl}_{3}\right) \delta 7.43(\mathrm{~d}, J=8.8 \mathrm{~Hz}, 4 \mathrm{H} ; o-t-\mathrm{Bu}-\mathrm{Ph}), 7.16(\mathrm{~d}, J=8.8 \mathrm{~Hz}, 4 \mathrm{H} ; m-t-\mathrm{Bu}-\mathrm{Ph})$, $6.86\left(\mathrm{~d}, J=1.8 \mathrm{~Hz}, 4 \mathrm{H} ; 4,6\right.$-orc), 6.62 (s, 2H; 2-orc), 5.04 (s, 2H; 5-pyr), 2.35 (s, 6H; $\left.\mathrm{CH}_{3}\right), 1.34$ (s, $18 \mathrm{H} ; t$-Bu); ${ }^{13} \mathrm{C}$ NMR $\left(75 \mathrm{MHz}, \mathrm{CDCl}_{3}\right) \delta 174.2$ (C; 4,6-pyr), 166.1 (C; 2-pyr), 152.8 (C; 1,3-orc), $150.0(\mathrm{C} ; \mathrm{Ph}), 148.4(\mathrm{C} ; \mathrm{Ph}), 143.7(\mathrm{C} ; 5$-orc), $126.7(\mathrm{CH} ; \mathrm{Ph}), 121.0(\mathrm{CH} ; \mathrm{Ph}), 120.7$ (CH; 4,6-orc), 111.9 (CH; 2-orc), $82.1\left(\mathrm{CH} ; 5\right.$-pyr), $34.6(\mathrm{C} ; t-\mathrm{Bu}), 31.6\left(\mathrm{CH}_{3} ; t-\mathrm{Bu}\right), 21.6\left(\mathrm{CH}_{3} ;\right.$ orc $)$.

4,6,16,18-tetraaza-11,23-dimethyl-5,17-bis(benzyloxy)-2,8,14,20-tetraoxacalix[4]arene (7c) (general procedure 4): To a stirred suspension of oxacalix[4]arene $4(50 \mathrm{mg}, 0.09 \mathrm{mmol})$ and $\mathrm{NaH}(60 \%$ dispersion; $11 \mathrm{mg}, 0.28 \mathrm{mmol})$ in $\mathrm{CH}_{3} \mathrm{CN}(3 \mathrm{~mL})$, benzyl alcohol $(6 \mathrm{cc})(21 \mu \mathrm{L}, 0.20 \mathrm{mmol}, 2.2$ equiv) was added, and the resulting mixture was stirred at $70{ }^{\circ} \mathrm{C}$ during 15 min (under an Ar atmosphere). Subsequently, the mixture was diluted with ethyl acetate $(50 \mathrm{~mL})$ and washed with distilled water $(3 \times$ $25 \mathrm{~mL}$ ). The organic fraction was dried over $\mathrm{MgSO}_{4}$, filtered and the solvent was removed under vacuum. After flash column chromatographic purification (silica, eluent heptane-ethyl acetate 4-1), oxacalix[4]arene 7c (36 mg, 65\%) was isolated as a white solid. mp 253-254 ${ }^{\circ} \mathrm{C}$; MS (APCI+) $\mathrm{m} / \mathrm{z}$ $613.6\left[\mathrm{MH}^{+}\right]$; HRMS (EI) calcd for $\mathrm{C}_{36} \mathrm{H}_{28} \mathrm{~N}_{4} \mathrm{O}_{6}$ : 612.2009; found: $m / z$ 612.2024; ${ }^{1} \mathrm{H}$ NMR (300 MHz, $\left.\mathrm{CDCl}_{3}\right) \delta 7.51(\mathrm{~d}, J=7.3 \mathrm{~Hz}, 4 \mathrm{H} ; o-\mathrm{Bn}), 7.45-7.30(\mathrm{~m}, 6 \mathrm{H} ; m, p-\mathrm{Bn}), 6.88$ (s, 4H; 4,6-orc), 6.62 (s, 2H; 2-orc), 5.48 (s, 4H; $\mathrm{CH}_{2}$ ), 5.02 (s, 2H; 5-pyr), $2.36\left(\mathrm{~s}, 6 \mathrm{H} ; \mathrm{CH}_{3}\right) ;{ }^{13} \mathrm{C} \mathrm{NMR}\left(75 \mathrm{MHz}, \mathrm{CDCl}_{3}\right) \delta 174.0$ (C; 4,6-pyr), 166.0 (C; 2-pyr), 152.9 (C; 1,3-orc), 143.7 (C; 5-orc), 136.0 (C; i-Bn), 128.6 (CH; Bn), 128.2 (CH; p-Bn), 128.1 (CH; Bn), 120.6 (CH; 4,6-orc), $111.9(\mathrm{CH} ; 2$-orc), 81.4 (CH; 5-pyr), 69.8 $\left(\mathrm{CH}_{2}\right), 21.6\left(\mathrm{CH}_{3}\right)$.

4,6,16,18-tetraaza-11,23-dimethyl-5,17-diethoxy-2,8,14,20-tetraoxacalix[4]arene (7d): Synthesis according to general procedure 4: oxacalix[4]arene $4(50 \mathrm{mg}, 0.09 \mathrm{mmol})$, ethanol (6d) $(13 \mu \mathrm{L}, 0.22$ mmol, 2.4 equiv), $\mathrm{NaH}(60 \%$ dispersion; $11 \mathrm{mg}, 0.28 \mathrm{mmol}), \mathrm{CH}_{3} \mathrm{CN}(3 \mathrm{~mL}), 70{ }^{\circ} \mathrm{C}, 20 \mathrm{~min}$; Eluent heptane-ethyl acetate 4-1; Yield 57\% (25 mg); mp 253-254 ${ }^{\circ} \mathrm{C}$; MS (APCI+) m/z $489.3\left[\mathrm{MH}^{+}\right.$]; HRMS (EI) calcd for $\mathrm{C}_{26} \mathrm{H}_{24} \mathrm{~N}_{4} \mathrm{O}_{6}$ : 488.1696; found: $\mathrm{m} / \mathrm{z}$ 488.1700; ${ }^{1} \mathrm{H}$ NMR (300 MHz, $\left.\mathrm{CDCl}_{3}\right) \delta 6.88(\mathrm{~d}, J=$ $1.8 \mathrm{~Hz}, 4 \mathrm{H} ; 4,6$-orc), 6.62 (s, 2H; 2-orc), 4.99 (s, 2H; 5-pyr), 4.47 (q, J=7.3 Hz, 4H; CH ), 2.37 (s, 6H; $\mathrm{CH}_{3}$-orc), $1.45\left(\mathrm{t}, J=7.3 \mathrm{~Hz}, 6 \mathrm{H} ; \mathrm{CH}_{3}\right.$-ethoxy); ${ }^{13} \mathrm{C} \mathrm{NMR}\left(75 \mathrm{MHz}, \mathrm{CDCl}_{3}\right) \delta 174.0(\mathrm{C} ; 4,6-$ pyr$), 166.2$ (C; 2-pyr), 152.9 (C; 1,3-orc), 143.6 (C; 5-orc), 120.5 (CH; 4,6-orc), 111.9 (CH; 2-orc), 81.1 (CH; 5pyr), $64.3\left(\mathrm{CH}_{2}\right), 21.6\left(\mathrm{CH}_{3} ;\right.$ orc $), 14.5\left(\mathrm{CH}_{3}\right.$; ethoxy $)$.

4,6,16,18-tetraaza-11,23-dimethyl-5,17-bis(thiophenoxy)-2,8,14,20-tetraoxacalix[4]arene Synthesis according to general procedure 3: oxacalix[4] arene $4(50 \mathrm{mg}, 0.09 \mathrm{mmol})$, thiophenol (6e) $(20$ $\mathrm{mg}, 0.18 \mathrm{mmol}), \mathrm{K}_{2} \mathrm{CO}_{3}$ (31 mg, $\left.0.22 \mathrm{mmol}\right), 18$-crown-6 (10 mg, $\left.0.04 \mathrm{mmol}\right), \mathrm{DMF}(3 \mathrm{~mL}), 70{ }^{\circ} \mathrm{C}, 48$ $\mathrm{h}$; Eluent heptane-ethyl acetate 4-1; Yield 95\% (53 mg); mp 134-135 ${ }^{\circ} \mathrm{C}$; MS (APCI+) $\mathrm{m} / \mathrm{z} 617.6$ [MH ${ }^{+}$; HRMS (EI) calcd for $\mathrm{C}_{34} \mathrm{H}_{24} \mathrm{~N}_{4} \mathrm{O}_{4} \mathrm{~S}_{2}: 616.1239$; found: $m / z$ 616.1239; ${ }^{1} \mathrm{H}$ NMR (400 MHz, $\left.\mathrm{CDCl}_{3}\right) \delta 7.68-7.63(\mathrm{~m}, 4 \mathrm{H} ; \mathrm{Ph}), 7.47-7.40(\mathrm{~m}, 6 \mathrm{H} ; \mathrm{Ph}), 6.82(\mathrm{~d}, J=1.5 \mathrm{~Hz}, 4 \mathrm{H} ; 4,6-\mathrm{orc}), 6.53(\mathrm{~s}, 2 \mathrm{H}$; 2-orc), $5.00\left(\mathrm{~s}, 2 \mathrm{H} ; 5\right.$-pyr), 2.32 (s, 6H; $\mathrm{CH}_{3}$-orc); ${ }^{13} \mathrm{C} \mathrm{NMR}\left(100 \mathrm{MHz}, \mathrm{CDCl}_{3}\right) \delta 174.6$ (C; 2-pyr), 172.4 (C; 4,6-pyr), 152.8 (C; 1,3-orc), 143.8 (C; 5-orc), $135.4(\mathrm{CH} ; \mathrm{Ph}), 129.6(\mathrm{CH} ; p$-Ph), $129.4(\mathrm{CH}$; $\mathrm{Ph}), 128.9(\mathrm{C} ; \mathrm{i}-\mathrm{Ph}), 120.6$ (CH; 4,6-orc), $111.7\left(\mathrm{CH} ; 2\right.$-orc), $83.6(\mathrm{CH} ; 5$-pyr $), 21.6\left(\mathrm{CH}_{3}\right)$.

4,6,16,18-tetraaza-11,23-dimethyl-5,17-bis(benzylmercapto)-2,8,14,20-tetraoxacalix[4]arene (7f): Synthesis according to general procedure 4: oxacalix[4]arene $4(50 \mathrm{mg}, 0.09 \mathrm{mmol})$, benzyl mercaptan (6f) $\left(24 \mu \mathrm{L}, 0.20 \mathrm{mmol}, 2.2\right.$ equiv), $\mathrm{NaH}(60 \%$ dispersion; $11 \mathrm{mg}, 0.28 \mathrm{mmol}), \mathrm{CH}_{3} \mathrm{CN}(3 \mathrm{~mL}), 70{ }^{\circ} \mathrm{C}$, $15 \mathrm{~min}$; Eluent heptane-ethyl acetate 4-1; Yield 72\% (42 mg); mp 100-101 ${ }^{\circ} \mathrm{C}$; MS (APCI+) $\mathrm{m} / \mathrm{z} 645.3$ [MH ${ }^{+}$; HRMS (EI) calcd for $\mathrm{C}_{36} \mathrm{H}_{28} \mathrm{~N}_{4} \mathrm{O}_{4} \mathrm{~S}_{2}$ : 644.1552; found: $\mathrm{m} / \mathrm{z}$ 644.1564; ${ }^{1} \mathrm{H}$ NMR $(300 \mathrm{MHz}$, $\left.\mathrm{CDCl}_{3}\right) \delta 7.46(\mathrm{~d}, J=6.4 \mathrm{~Hz}, 4 \mathrm{H} ; o-\mathrm{Bn}), 7.40-7.25(\mathrm{~m}, 6 \mathrm{H} ; m, p-\mathrm{Bn}), 6.88$ (s, 4H; 4,6-orc), 6.59 (s, 2H; 
2-orc), 5.04 (s, 2H; 5-pyr), $4.46\left(\mathrm{~s}, 4 \mathrm{H} ; \mathrm{CH}_{2}\right), 2.37\left(\mathrm{~s}, 6 \mathrm{H} ; \mathrm{CH}_{3}\right) ;{ }^{13} \mathrm{C} \mathrm{NMR}\left(75 \mathrm{MHz}, \mathrm{CDCl}_{3}\right) \delta 174.2$ (C; 2-pyr), 172.2 (C; 4,6-pyr), 152.8 (C; 1,3-orc), 143.9 (C; 5-orc), 136.8 (C; i-Bn), 129.4 (CH; Bn), 128.7 (CH; Bn), $127.5(\mathrm{CH} ;$ p-Bn), $120.6(\mathrm{CH} ; 4,6$-orc), 111.7 (CH; 2-orc), 83.2 (CH; 5-pyr), 35.7 $\left(\mathrm{CH}_{2}\right), 21.6\left(\mathrm{CH}_{3}\right)$.

4,6,16,18-tetraaza-11,23-dimethyl-5,17-bis(phenylamino)-2,8,14,20-tetraoxacalix[4]arene (7g): To a solution of oxacalix[4] arene $4(50 \mathrm{mg}, 0.09 \mathrm{mmol})$ in DMSO $(1 \mathrm{~mL})$, aniline $(\mathbf{6 g})(57 \mu \mathrm{L}, 0.45$ mmol, 7 equiv) was added, and the resulting mixture was heated at $170{ }^{\circ} \mathrm{C}$ for $4 \mathrm{~h}$ (under an $\mathrm{Ar}$ atmosphere). Subsequently, the mixture was cooled down to rt, distilled water $(25 \mathrm{~mL})$ was added and the mixture was extracted with ethyl acetate $(3 \times 25 \mathrm{~mL})$. The collected organic fractions were dried over $\mathrm{MgSO}_{4}$, filtered, and the solvent was evaporated in vacuum. After flash column chromatographic purification (silica, eluent heptane-ethyl acetate 4-1), oxacalix[4]arene $\mathbf{7 g}$ was isolated as a white solid (25 mg, 48\%). mp 175-176 ${ }^{\circ} \mathrm{C}$; MS (APCI+) m/z $583.4\left[\mathrm{MH}^{+}\right]$; HRMS (EI) calcd for $\mathrm{C}_{34} \mathrm{H}_{26} \mathrm{~N}_{6} \mathrm{O}_{4}$ : 582.2016; found: $\mathrm{m} / \mathrm{z} 582.2009 ;{ }^{1} \mathrm{H}$ NMR $\left(400 \mathrm{MHz}, \mathrm{CDCl}_{3}\right) \delta 7.63(\mathrm{~d}, J=7.8 \mathrm{~Hz}, 4 \mathrm{H} ; o-\mathrm{Ph}), 7.36(\mathrm{t}, J$ $=7.8 \mathrm{~Hz}, 4 \mathrm{H} ; m-\mathrm{Ph}), 7.19(\mathrm{~s}, 2 \mathrm{H}, \mathrm{NH}), 7.08(\mathrm{t}, J=7.3 \mathrm{~Hz}, 2 \mathrm{H} ; p-\mathrm{Ph}), 6.87$ (d, $J=1.5 \mathrm{~Hz}, 4 \mathrm{H} ; 4,6$-orc), 6.65 (s, 2H; 2-orc), 4.83 (s, 2H; 5-pyr), $2.36\left(\mathrm{~s}, 6 \mathrm{H} ; \mathrm{CH}_{3}\right) ;{ }^{13} \mathrm{C} \mathrm{NMR}\left(100 \mathrm{MHz}, \mathrm{CDCl}_{3}\right) \delta 173.2(\mathrm{C} ; 4,6-$ pyr), 160.6 (C; 2-pyr), 153.0 (C; 1,3-orc), 143.3 (C; 5-orc), 138.7 (C; i-Ph), $129.2(\mathrm{CH}), 123.5$ (CH; $p$ $\mathrm{Ph}), 120.5(\mathrm{CH}), 120.2(\mathrm{CH}), 112.3\left(\mathrm{CH} ; 2\right.$-orc), $79.1(\mathrm{CH} ; 5$-pyr $), 21.6\left(\mathrm{CH}_{3}\right)$.

4,6,16,18-tetraaza-11,23-dimethyl-5,17-bis(piperidinyl)-2,8,14,20-tetraoxacalix[4]arene (7h): To a solution of oxacalix[4] arene $4(50 \mathrm{mg}, 0.09 \mathrm{mmol})$ in 1,4-dioxane $(3 \mathrm{~mL})$, piperidine $(6 \mathrm{~h})(44 \mu \mathrm{L}, 0.44$ mmol, 5 equiv) was added, and the resulting mixture was heated at reflux for $2 \mathrm{~h}$ (under an $\mathrm{Ar}$ atmosphere). Subsequently, the mixture was cooled down to $\mathrm{rt}$, distilled water $(25 \mathrm{~mL})$ was added and the mixture was extracted with ethyl acetate $(3 \times 25 \mathrm{~mL})$. The collected organic fractions were dried over $\mathrm{MgSO}_{4}$, filtered, and the solvent was evaporated in vacuum. After flash column chromatographic purification (silica, eluent heptane-ethyl acetate 4-1), oxacalix[4]arene $7 \mathbf{h}$ was isolated as a white solid (34 mg, 67\%). mp 281-282 ${ }^{\circ} \mathrm{C}$; MS (APCI+) m/z $567.4\left[\mathrm{MH}^{+}\right]$; HRMS (EI) calcd for $\mathrm{C}_{32} \mathrm{H}_{34} \mathrm{~N}_{6} \mathrm{O}_{4}$ : 566.2642; found: $\mathrm{m} / \mathrm{z} 566.2680 ;{ }^{1} \mathrm{H} \mathrm{NMR}\left(400 \mathrm{MHz}, \mathrm{CDCl}_{3}\right) \delta 6.81$ (d, $J=1.5 \mathrm{~Hz}, 4 \mathrm{H} ; 4,6$-orc), 6.61 (s, 2H; 2-orc), 4.57 (s, 2H; 5-pyr), 3.86-3.79 (m, 8H; $\left.\mathrm{CH}_{2}\right), 2.33$ (s, 6H; $\left.\mathrm{CH}_{3}\right), 1.72-1.58(\mathrm{~m}, 12 \mathrm{H}$; $\left.\mathrm{CH}_{2}\right) ;{ }^{13} \mathrm{C}$ NMR $\left(75 \mathrm{MHz}, \mathrm{CDCl}_{3}\right) \delta 173.0(\mathrm{C} ; 4,6$-pyr), $161.6(\mathrm{C} ; 2$-pyr), 153.0 (C; 1,3-orc), $142.6(\mathrm{C}$; 5-orc), 120.1 (CH; 4,6-orc), $112.4\left(\mathrm{CH} ; 2\right.$-orc), 75.7 (CH; 5-pyr), 45.1 $\left(\mathrm{CH}_{2}\right), 26.0\left(\mathrm{CH}_{2}\right), 24.8\left(\mathrm{CH}_{2}\right)$, $21.5\left(\mathrm{CH}_{3}\right)$.

4,6,16,18-tetraaza-11,23-dimethyl-5,17-diamino-2,8,14,20-tetraoxacalix[4]arene (7i): Oxacalix[4]arene $4(50 \mathrm{mg}, 0.09 \mathrm{mmol})$ and ammonia (6i) $(2.0 \mathrm{M}$ solution in isopropanol, $1.5 \mathrm{~mL}, 3 \mathrm{mmol})$ were combined in a closed vessel and the resulting mixture was heated at $65{ }^{\circ} \mathrm{C}$ for $60 \mathrm{~h}$ (under an $\mathrm{Ar}$ atmosphere). Subsequently, the mixture was cooled down to $\mathrm{rt}$ and oxacalix[4]arene 7i was isolated as a white solid in pure form by simple filtration from the reaction mixture $(35 \mathrm{mg}, 91 \%) . \mathrm{mp}>325{ }^{\circ} \mathrm{C}$; MS (APCI+) $\mathrm{m} / \mathrm{z} 431.3\left[\mathrm{MH}^{+}\right.$]; HRMS (ESI) calcd for $\mathrm{C}_{22} \mathrm{H}_{18} \mathrm{~N}_{6} \mathrm{O}_{4}$ : 430.1390; found: $\mathrm{m} / \mathrm{z}$ 432.2371; ${ }^{1} \mathrm{H}$ NMR (300 MHz, DMSO-d $\left.{ }_{6}\right) \delta 6.94$ (s, 4H; 4,6-orc), 6.92 (s, 4H; $\left.\mathrm{NH}_{2}\right), 6.86$ (s, 2H; 2-orc), 4.49 (s, 2H; 5-pyr), 2.27 (s, 6H; $\left.\mathrm{CH}_{3}\right) ;{ }^{13} \mathrm{C}$ NMR (75 MHz, DMSO-d $) \delta 172.9$ (C; 4,6-pyr), 164.1 (C; 2-pyr), 153.0 (C; 1,3-orc), 143.4 (C; 5-orc), 120.2 (CH; 4,6-orc), 112.7 (CH; 2-orc), 77.0 (CH; 5-pyr), $21.1\left(\mathrm{CH}_{3}\right)$.

4,6,16,18-tetraaza-11,23-dimethyl-5,17-bis[di(ethoxycarbonyl)methyl]-2,8,14,20-tetraoxacalix[4]arene (7j): Synthesis according to general procedure 4: oxacalix[4]arene 4 (50 $\mathrm{mg}, 0.09 \mathrm{mmol})$, diethyl malonate $(6 \mathbf{j})\left(30 \mu \mathrm{L}, 0.20 \mathrm{mmol}, 2.2\right.$ equiv), $\mathrm{NaH}(60 \%$ dispersion; $11 \mathrm{mg}, 0.28 \mathrm{mmol}), \mathrm{CH}_{3} \mathrm{CN}$ $(3 \mathrm{~mL}), 70{ }^{\circ} \mathrm{C}, 15 \mathrm{~min}$; Eluent heptane-ethyl acetate 4-1; Yield 67\% (43 mg); mp 219-220 ${ }^{\circ} \mathrm{C}$; MS (APCI+) $\mathrm{m} / \mathrm{z} 717.8\left[\mathrm{MH}^{+}\right.$]; HRMS (EI) calcd for $\mathrm{C}_{36} \mathrm{H}_{36} \mathrm{~N}_{4} \mathrm{O}_{12}$ : 716.2330; found: $\mathrm{m} / \mathrm{z} 716.2359 ;{ }^{1} \mathrm{H}$ NMR (300 MHz, $\left.\mathrm{CDCl}_{3}\right) \delta 6.92$ (s, 4H; 4,6-orc), 6.63 (s, 2H; 2-orc), 5.37 (s, 2H; 5-pyr), 4.99 (s, 2H; $\mathrm{CH}), 4.31$ (q, $\left.J=7.3 \mathrm{~Hz}, 8 \mathrm{H} ; \mathrm{CH}_{2}\right), 2.39$ (s, $6 \mathrm{H} ; \mathrm{CH}_{3}$-orc), $1.32\left(\mathrm{t}, J=7.3 \mathrm{~Hz}, 12 \mathrm{H} ; \mathrm{CH}_{3}\right.$-ethyl); ${ }^{13} \mathrm{C}$ NMR (75 MHz, $\left.\mathrm{CDCl}_{3}\right) \delta 172.9$ (C), 166.0 (C), 165.1 (C), 152.7 (C; 1,3-orc), 144.2 (C; 5-orc), 120.8 (CH; 4,6-orc), $111.6\left(\mathrm{CH} ; 2\right.$-orc), $86.2\left(\mathrm{CH} ; 5\right.$-pyr), $62.4\left(\mathrm{CH}_{2}\right), 61.5(\mathrm{CH}), 21.6\left(\mathrm{CH}_{3} ;\right.$ orc $), 14.2\left(\mathrm{CH}_{3}\right.$; ethyl).

1,8-Bis(2-hydroxyphenoxy)-3,6-dioxaoctane (6k): This compound has been prepared before by Stoddart et al. Synthesis of $\mathbf{6 k}$ has been performed according to a procedure described by Reinhoudt et

${ }^{3}$ Colquhoun, H. M.; Goodings, E. P.; Maud, J. M.; Stoddart, J. F.; Wolstenholme, J. B.; Williams, D. J. J. Chem. Soc., Perkin Trans. 2 1985, 607. 
al. (for an analogous product). ${ }^{4}$ Material identity and purity was confirmed by $\mathrm{MS}(\mathrm{CI}),{ }^{1} \mathrm{H}$ and ${ }^{13} \mathrm{C}$ NMR.

\section{4,6,16,18-tetraaza-11,23-dimethyl-5,17-[2,2'-(3,6-dioxaoctane-1,8-diyldioxy)bis(phenoxy)]-}

2,8,14,20-tetraoxacalix[4] arene (7k): $\mathrm{K}_{2} \mathrm{CO}_{3}(62 \mathrm{mg}, 0.45 \mathrm{mmol})$ and 18 -crown-6 (10 mg, $\left.0.04 \mathrm{mmol}\right)$ were mixed in DMF $(150 \mathrm{~mL})$ and stirred at $70{ }^{\circ} \mathrm{C}$ (under an Ar atmosphere). Oxacalix[4]arene 4 (100 $\mathrm{mg}, 0.18 \mathrm{mmol}, 1$ equiv) and bisphenol $6 \mathbf{k}(60 \mathrm{mg}, 0.18 \mathrm{mmol}, 1$ equiv) were dissolved separately in 10 $\mathrm{mL}$ DMF, and subsequently added dropwise (equimolarly) to the stirring mixture over $12 \mathrm{~h}$ using a syringe pump. The resulting mixture was then stirred for another $24 \mathrm{~h}$ at $70{ }^{\circ} \mathrm{C}$. DMF was evaporated in vacuum and the crude residue was redissolved in ethyl acetate $(100 \mathrm{~mL})$ and washed with distilled water $(3 \times 50 \mathrm{~mL})$. The organic fraction was dried over $\mathrm{MgSO}_{4}$, filtered and the solvent was removed under vacuum. After flash column chromatographic purification (silica, eluent $\mathrm{CH}_{2} \mathrm{Cl}_{2}$-ethyl acetate 9-1), oxacalixcrown $7 \mathbf{k}$ was isolated as a white solid $(101 \mathrm{mg}, 77 \%)$. $\mathrm{mp} 143-144{ }^{\circ} \mathrm{C}$; MS (APCI + ) $\mathrm{m} / \mathrm{z} 731.6$ [MH ${ }^{+}$; HRMS (EI) calcd for $\mathrm{C}_{40} \mathrm{H}_{34} \mathrm{~N}_{4} \mathrm{O}_{10}$ : 730.2275; found: $m / z$ 730.2265; ${ }^{1} \mathrm{H}$ NMR $(300 \mathrm{MHz}$, $\left.\mathrm{CDCl}_{3}\right) \delta 7.26-7.17$ (m, 4H; cat), 7.04-6.93 (m, 4H; cat), 6.84 (d, J=1.8 Hz, 4H; 4,6-orc), 6.54 (s, 2H; 2-orc), 4.93 (s, 2H; 5-pyr), 4.14-4.05 (m, 4H; $\left.\mathrm{CH}_{2}\right), 3.76-3.70\left(\mathrm{~m}, 4 \mathrm{H} ; \mathrm{CH}_{2}\right), 3.35\left(\mathrm{~s}, 4 \mathrm{H} ; \mathrm{CH}_{2}\right), 2.34(\mathrm{~s}$, 6H; $\left.\mathrm{CH}_{3}\right) ;{ }^{13} \mathrm{C}$ NMR (75 MHz, $\left.\mathrm{CDCl}_{3}\right) \delta 174.2$ (C; 4,6-pyr), 166.1 (C; 2-pyr), 152.9 (C; 1,3-orc), 150.4 (C; cat), 143.7 (C; 5-orc), $141.9(\mathrm{C}$; cat), 126.7 (CH; cat), $122.3(\mathrm{CH}$; cat), $121.5(\mathrm{CH}$; cat), $120.6(\mathrm{CH}$; 4,6-orc), 113.4 (CH; cat), 111.9 (CH; 2-orc), 81.9 (CH; 5-pyr), $70.9\left(\mathrm{CH}_{2}\right), 70.1\left(\mathrm{CH}_{2}\right), 68.5\left(\mathrm{CH}_{2}\right), 21.6$ $\left(\mathrm{CH}_{3}\right)$.

\section{4,6,16,18-tetraaza-11,23-dimethyl-5,17-bis[(S)-2-amino-2-(ethoxycarbonyl)ethylsulfanyl]-}

2,8,14,20-tetraoxacalix[4]arene (7l): Oxacalix[4]arene $4(50 \mathrm{mg}, 0.09 \mathrm{mmol})$, L-cysteine ethyl ester hydrochloride (6l) $\left(34 \mathrm{mg}, 0.23 \mathrm{mmol}, 2.5\right.$ equiv), and $\mathrm{Et}_{3} \mathrm{~N}(50 \mu \mathrm{L}, 0.36 \mathrm{mmol})$ were dissolved in $\mathrm{CH}_{3} \mathrm{CN}(3 \mathrm{~mL})$ and the resulting mixture was stirred at $70{ }^{\circ} \mathrm{C}$ during 90 min (under an $\mathrm{Ar}$ atmosphere). Subsequently, the mixture was cooled down to $\mathrm{rt}$, distilled water was added $(25 \mathrm{~mL})$ and the mixture was extracted with ethyl acetate $(3 \times 25 \mathrm{~mL})$, The collected organic fractions were dried over $\mathrm{MgSO}_{4}$, filtered, and the solvent was evaporated in vacuum. After flash column chromatographic purification (silica, eluent $\mathrm{CH}_{2} \mathrm{Cl}_{2}$-ethyl acetate 1-1), oxacalix[4]arene 7l was isolated as a white solid (44 mg, 71\%). mp 144-145 ${ }^{\circ} \mathrm{C}$; MS (APCI+) m/z 695.2 [MH ${ }^{+}$]; HRMS (ESI) calcd for $\mathrm{C}_{32} \mathrm{H}_{34} \mathrm{~N}_{6} \mathrm{O}_{8} \mathrm{~S}_{2}: 694.1880$; found: $\mathrm{m} / \mathrm{z} 695.1939\left[\mathrm{MH}^{+}\right]$; ${ }^{1} \mathrm{H}$ NMR $\left(300 \mathrm{MHz}, \mathrm{CDCl}_{3}\right) \delta 6.88$ (s, 4H; 4,6-orc), 6.58 (s, 2H; 2-orc), 5.03 (s, 2H; 5-pyr), 4.22 (q, $J=7.3 \mathrm{~Hz}, 4 \mathrm{H} ; \mathrm{CH}_{2}$-ethyl), 3.93-3.87 (m, 2H; CH), 3.71-3.62 (dd, $J=$ $\left.13.7,4.6 \mathrm{~Hz}, 2 \mathrm{H} ; \mathrm{CH}_{2}-\mathrm{S}\right), 3.51-3.42\left(\mathrm{dd}, J=13.7,6.4 \mathrm{~Hz}, 2 \mathrm{H} ; \mathrm{CH}_{2}-\mathrm{S}\right), 2.37$ (s, 6H; $\mathrm{CH}_{3}$-orc), 2.00 (s, $\left.4 \mathrm{H}, \mathrm{NH}_{2}\right), 1.31\left(\mathrm{t}, J=7.3 \mathrm{~Hz}, 6 \mathrm{H} ; \mathrm{CH}_{3}\right.$-ethyl); ${ }^{13} \mathrm{C} \mathrm{NMR}\left(100 \mathrm{MHz}, \mathrm{CDCl}_{3}\right) \delta 173.7(\mathrm{C} ; 2$-pyr $), 173.5$ (C; CO), 172.2 (C; 4,6-pyr), 152.8 (C; 1,3-orc), 143.9 (C; 5-orc), 120.6 (CH; 4,6-orc), 111.6 (CH; 2orc), $83.5\left(\mathrm{CH} ; 5\right.$-pyr), $61.6\left(\mathrm{CH}_{2}\right.$; ethyl), $54.1(\mathrm{CH}), 35.6\left(\mathrm{CH}_{2} ; \mathrm{CH}_{2}-\mathrm{S}\right), 21.6\left(\mathrm{CH}_{3} ;\right.$ orc $), 14.3\left(\mathrm{CH}_{3}\right.$; ethyl).

4,4'-bis(4,6,16,18-tetraaza-11,23-dimethyl-2,8,14,20-tetraoxacalix[4]arene-5-yloxy)biphenyl (9): Synthesis according to general procedure 3: mono(methylsulfonyl)oxacalix[4]arene 5 (50 mg, 0.104 mmol, 2 equiv), 4,4'-biphenol (8) (10 mg, $0.054 \mathrm{mmol}$, 1 equiv), $\mathrm{K}_{2} \mathrm{CO}_{3}$ (22 mg, $0.16 \mathrm{mmol}$ ), 18 crown-6 (5 mg, $0.02 \mathrm{mmol})$, DMF $(3 \mathrm{~mL}), 70{ }^{\circ} \mathrm{C}, 24 \mathrm{~h}$; Eluent heptane-ethyl acetate 1-1; Yield $62 \%$ (32 mg); mp 218-219 ${ }^{\circ} \mathrm{C}$; MS (APCI+) $\mathrm{m} / \mathrm{z} 983.4\left[\mathrm{MH}^{+}\right]$; HRMS (MALDI) calcd for $\mathrm{C}_{56} \mathrm{H}_{38} \mathrm{~N}_{8} \mathrm{O}_{10}$ : 982.2711; found: $\mathrm{m} / \mathrm{z} 983.3542[\mathrm{M}+\mathrm{H}]^{+}, 1005.3363[\mathrm{M}+\mathrm{Na}]^{+} ;{ }^{1} \mathrm{H}$ NMR $\left(400 \mathrm{MHz}, \mathrm{CDCl}_{3}\right) \delta 8.68(\mathrm{~s}$, 2H; 2-pyr), 7.62 (d, $J=8.6 \mathrm{~Hz}, 4 \mathrm{H}$; Ph), 7.31 (d, $J=8.6 \mathrm{~Hz}, 4 \mathrm{H} ; \mathrm{Ph}), 6.90$ (s, 8H; 4,6-orc), 6.64 (s, 4H; 2-orc), 5.42 (s, 2H; 5-pyr), 5.09 (s, 2H; 5-pyr), $2.37\left(\mathrm{~s}, 12 \mathrm{H} ; \mathrm{CH}_{3}\right) ;{ }^{13} \mathrm{C}$ NMR $\left(100 \mathrm{MHz}, \mathrm{CDCl}_{3}\right) \delta$ 174.3 (C; 4/6-pyr), 172.8 (C; 4/6-pyr), 166.1 (C; 2-pyr), 160.3 (CH; 2-pyr), 153.0 (C; 1/3-orc), 152.8 (C; 1/3-orc), 151.9 (C), 144.1 (C; 5-orc), $138.3(\mathrm{C}), 128.6(\mathrm{CH}), 122.1(\mathrm{CH}), 120.8$ (CH; 4,6-orc), 120.7 (CH; 4,6-orc), 111.7 (CH; 2-orc), 87.7 (CH; 5-pyr), 82.4 (CH; 5-pyr), $21.6\left(\mathrm{CH}_{3}\right)$. 
3. ${ }^{1} \mathrm{H}$ and ${ }^{13} \mathrm{C}$ NMR spectra for oxacalix[2]arene[2]pyrimidines 3a-d, 4, 5, 7a-l, and 9
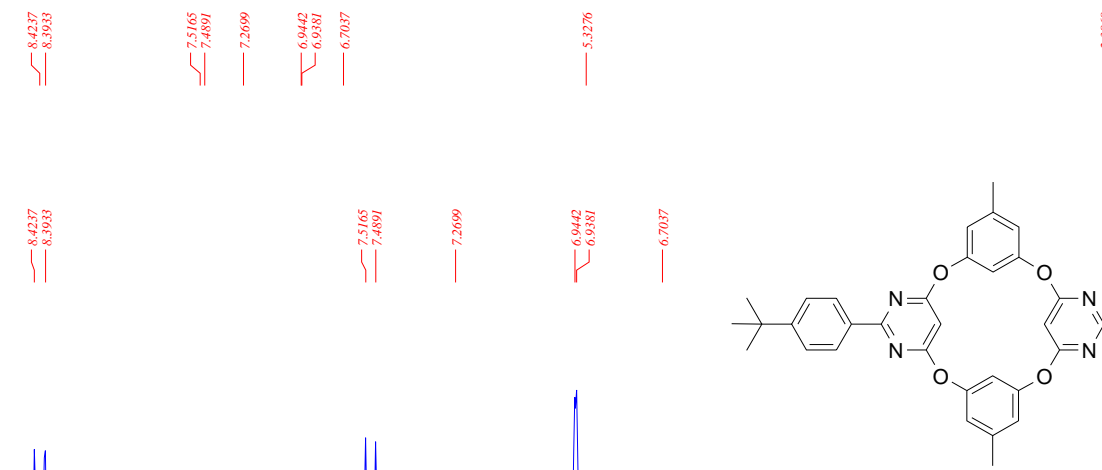

$3 a$
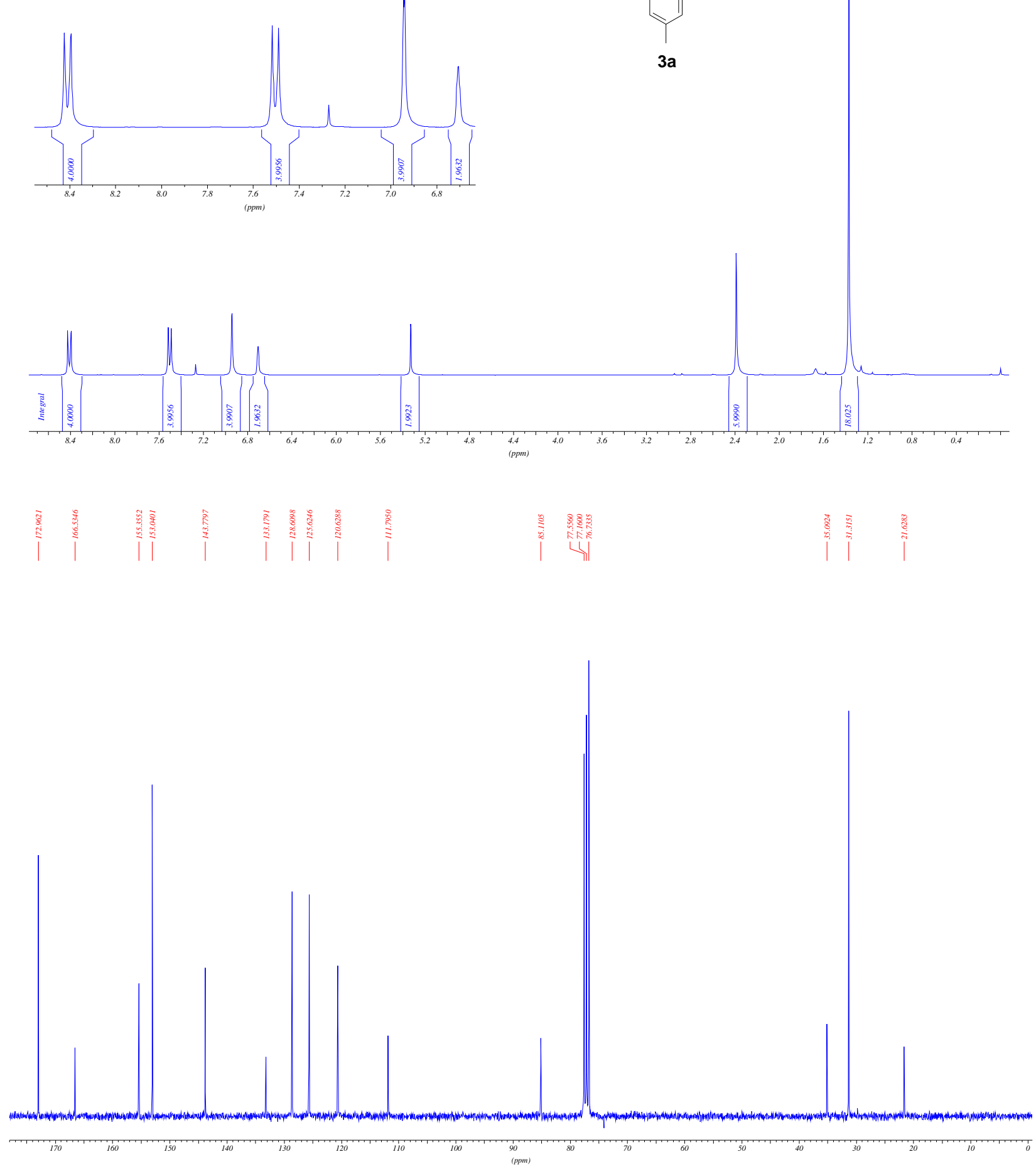

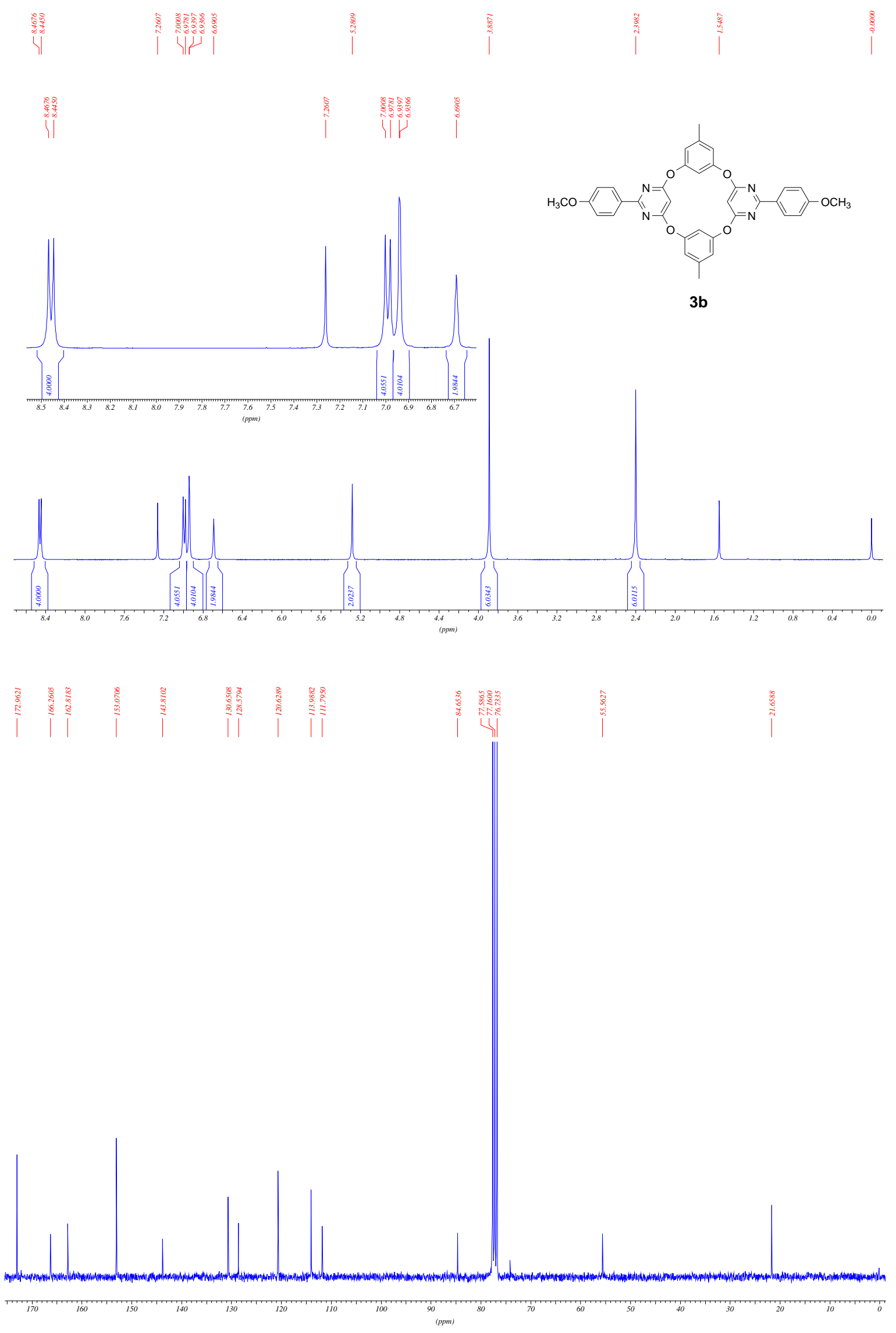

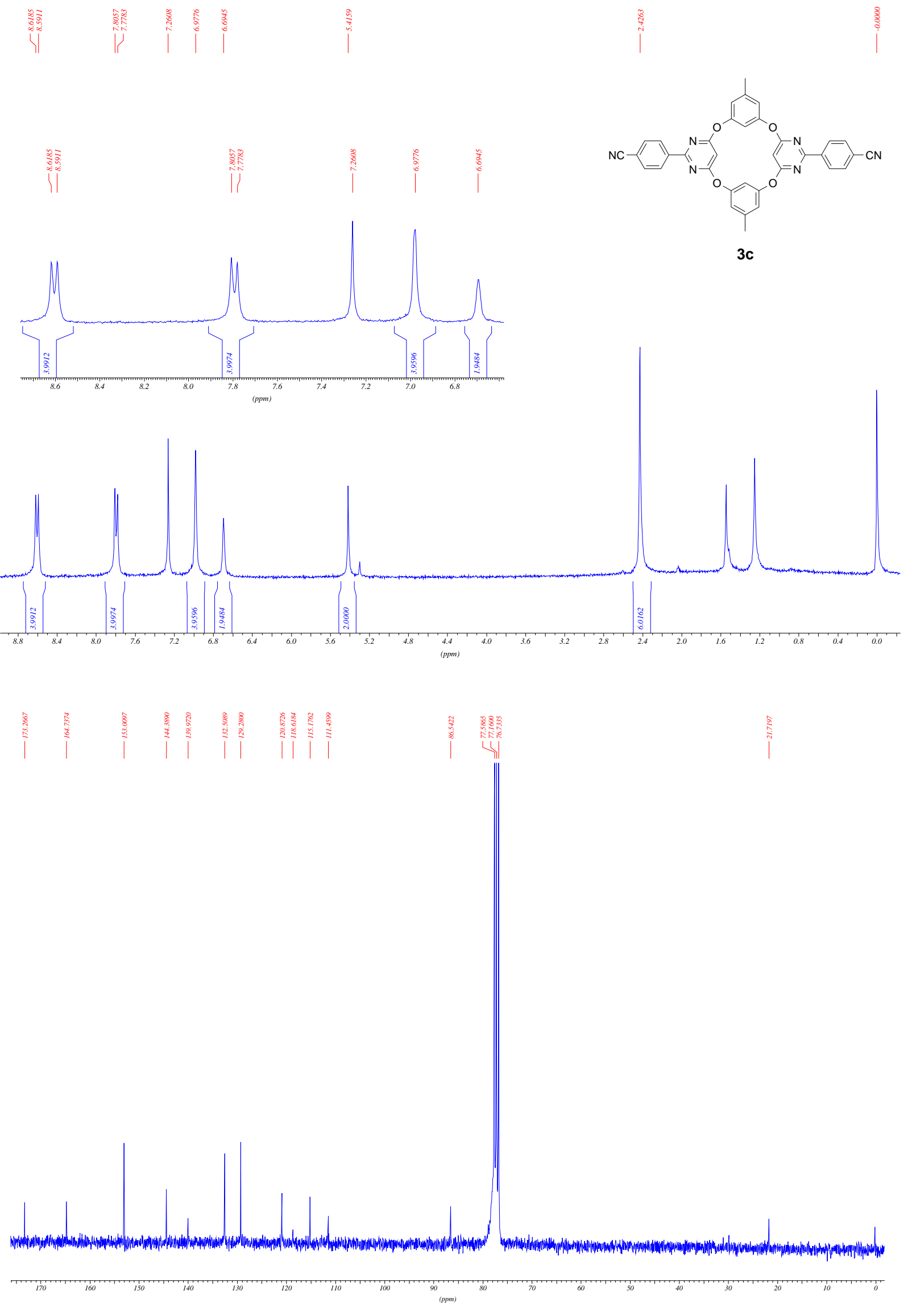


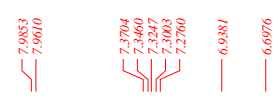
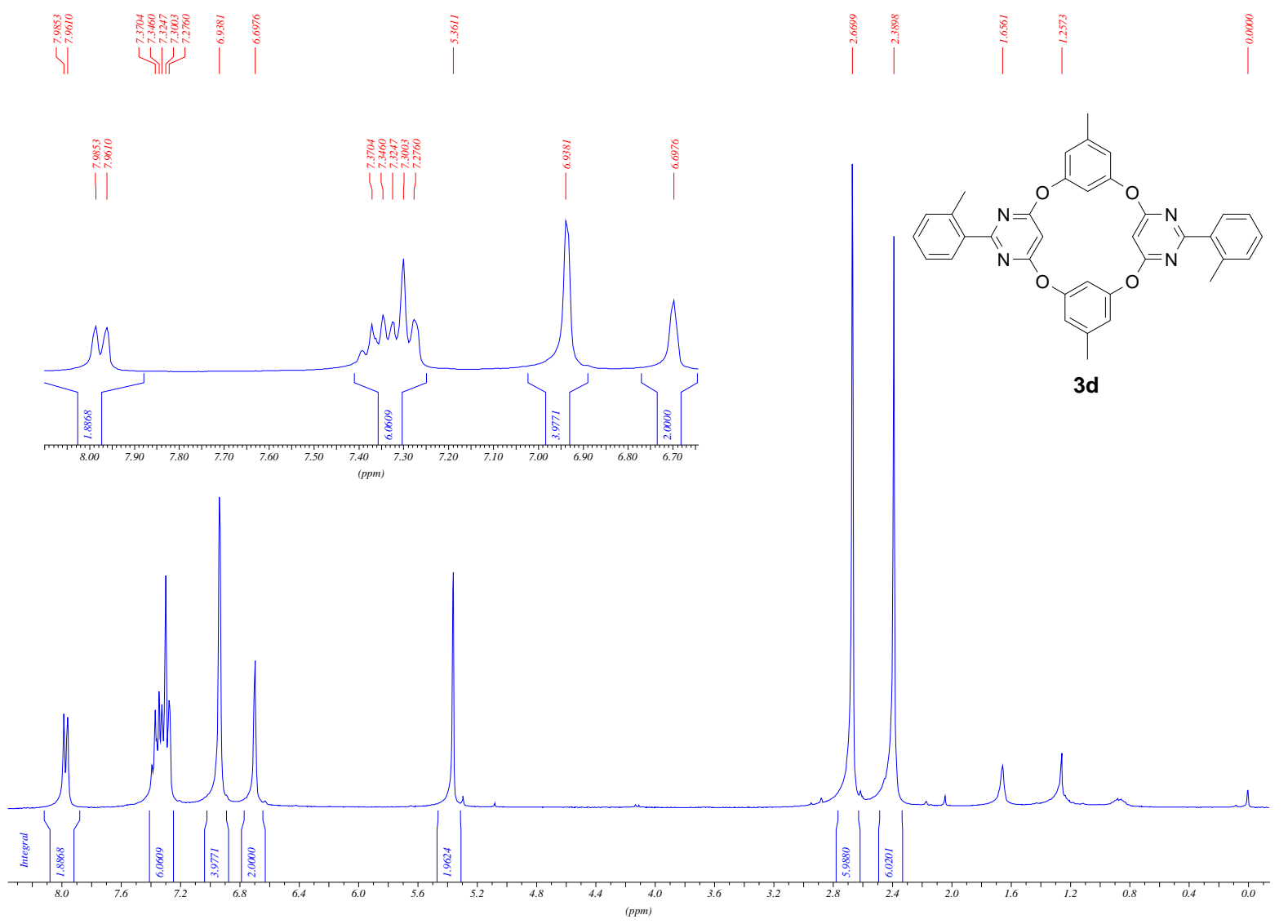

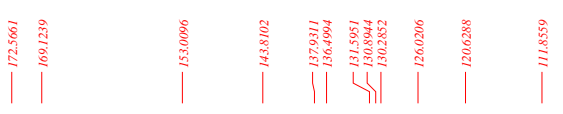

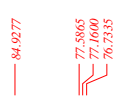

3d

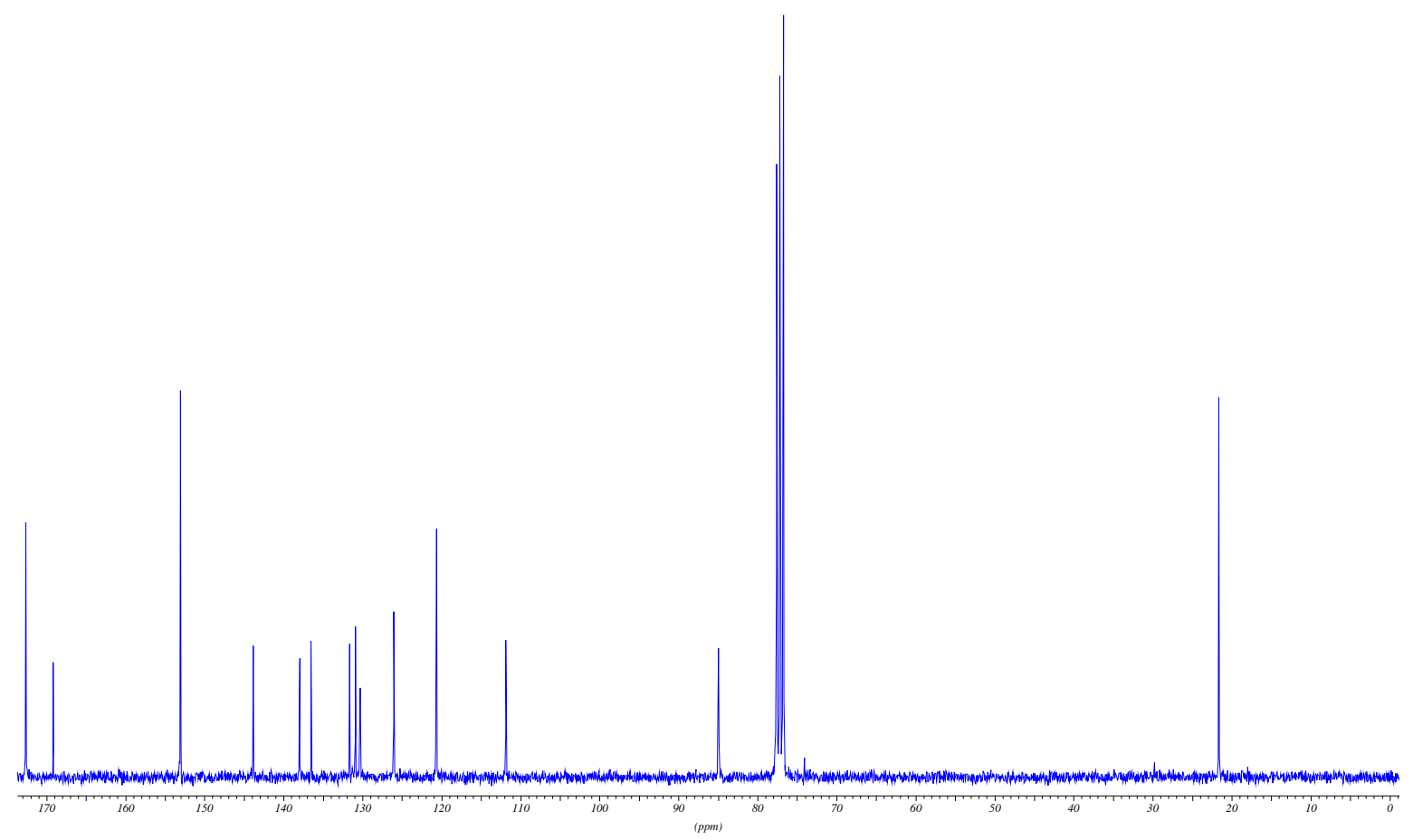




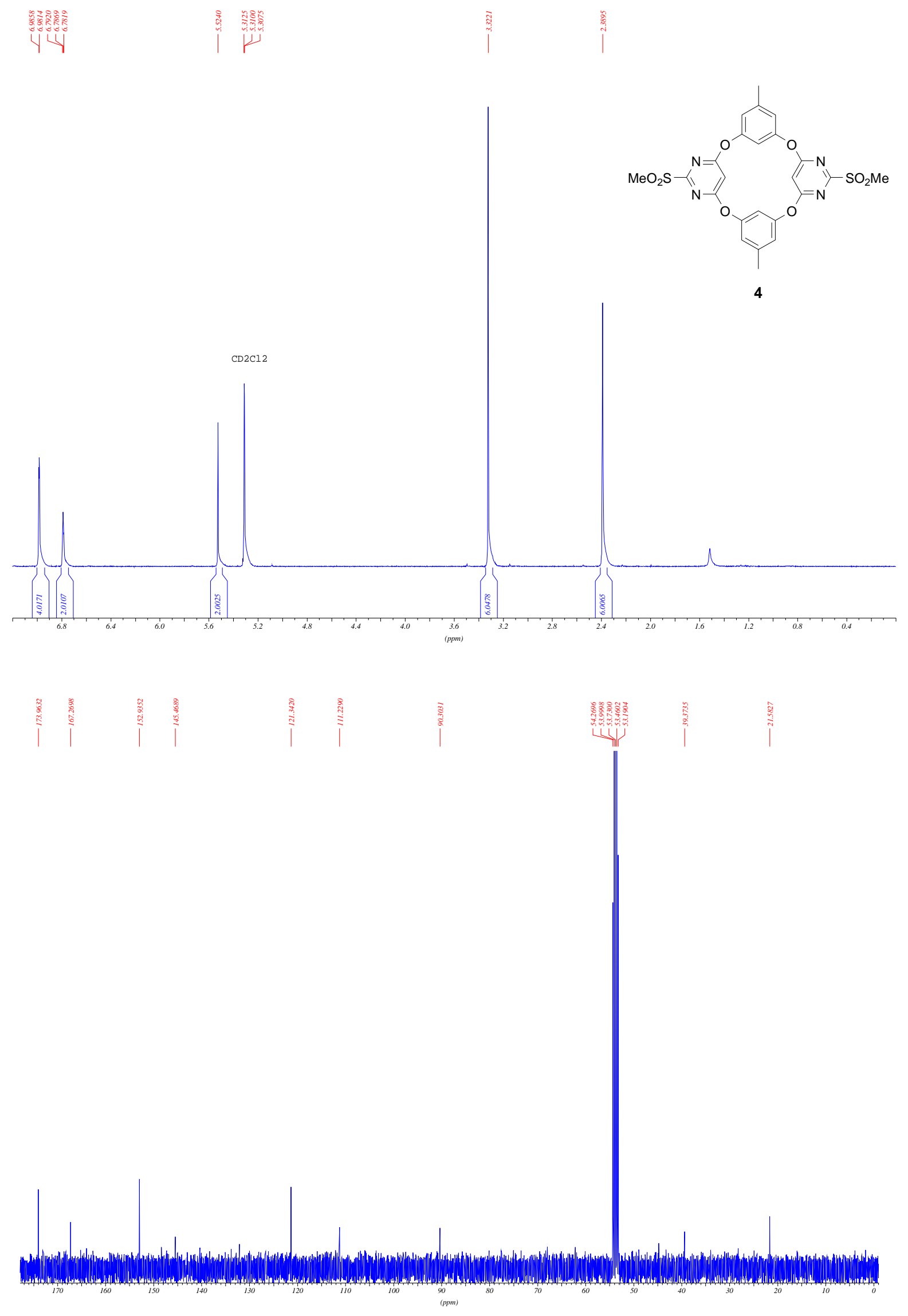



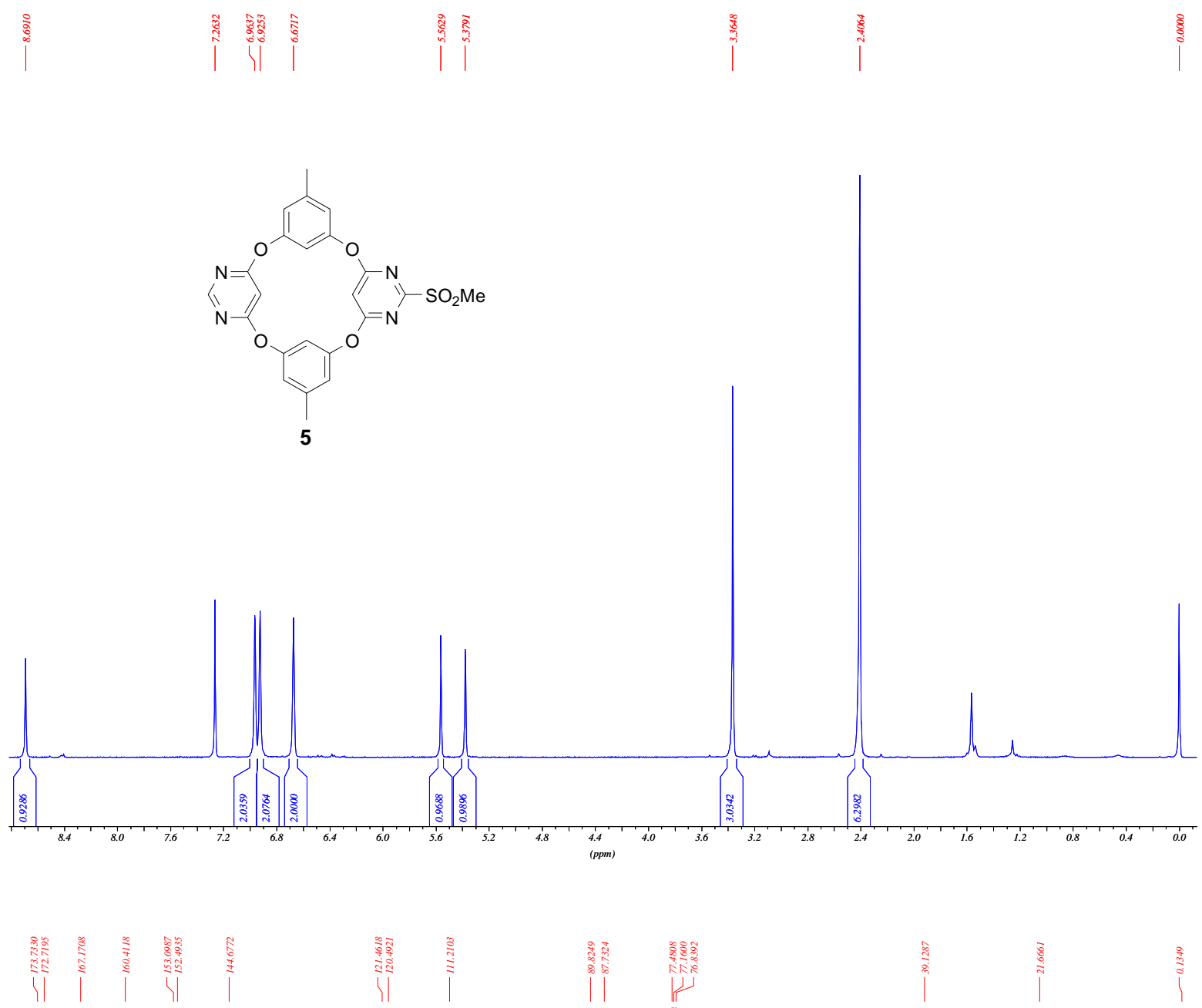


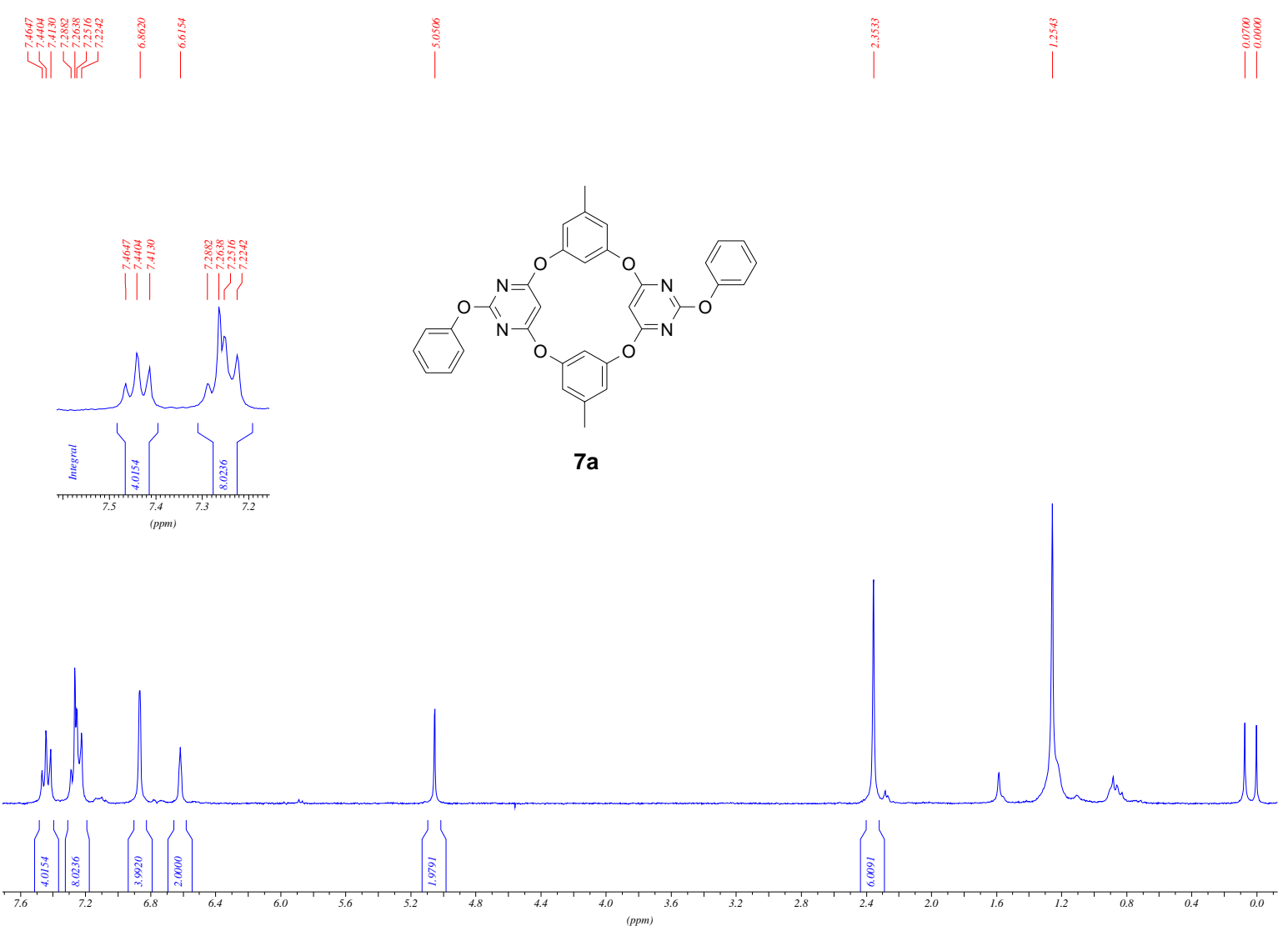

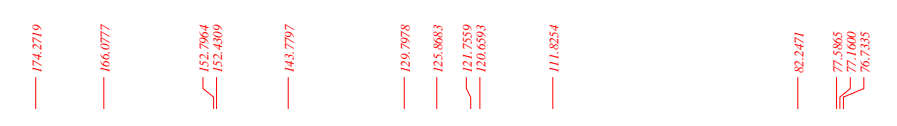

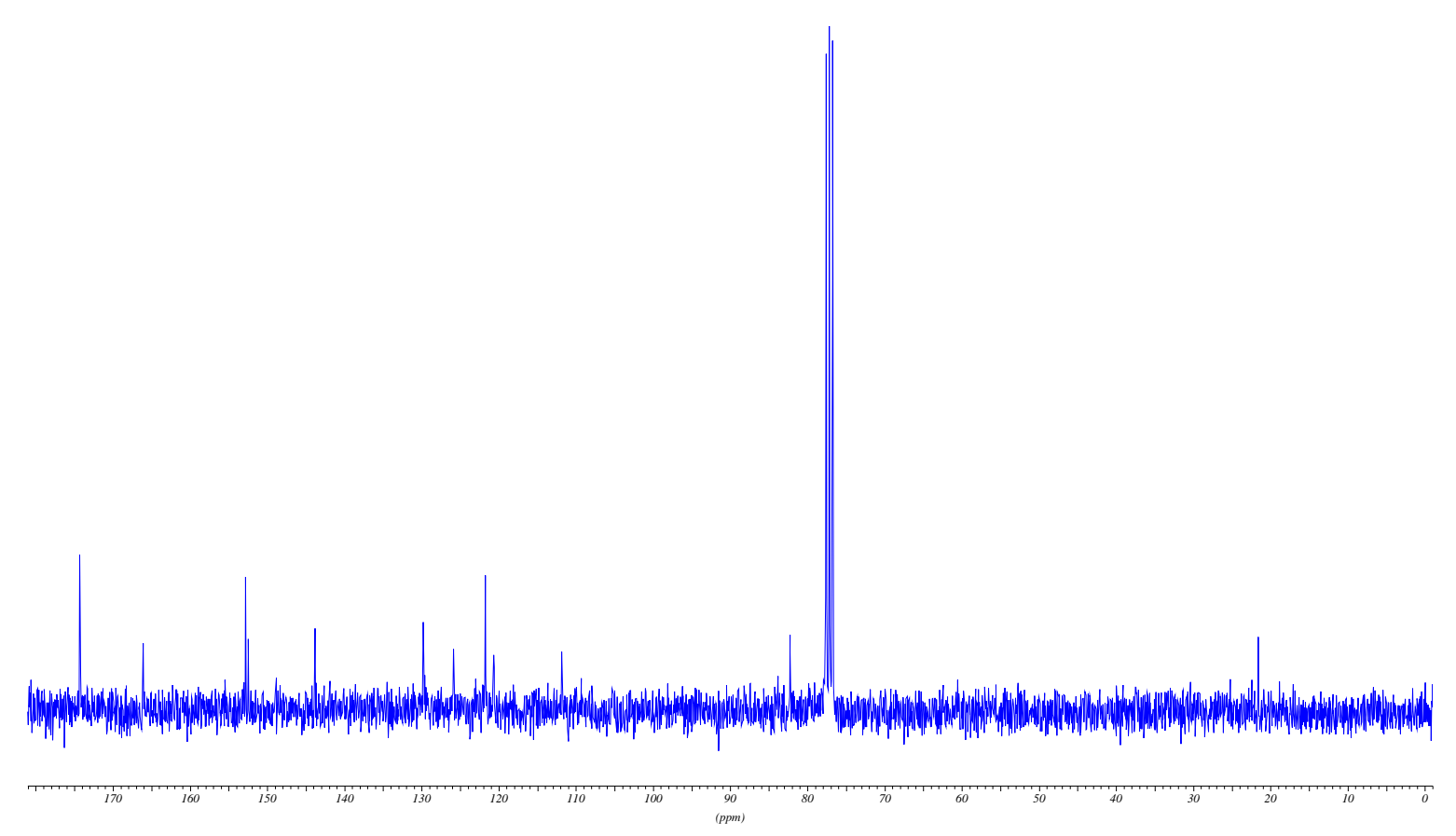




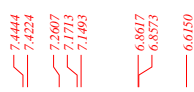

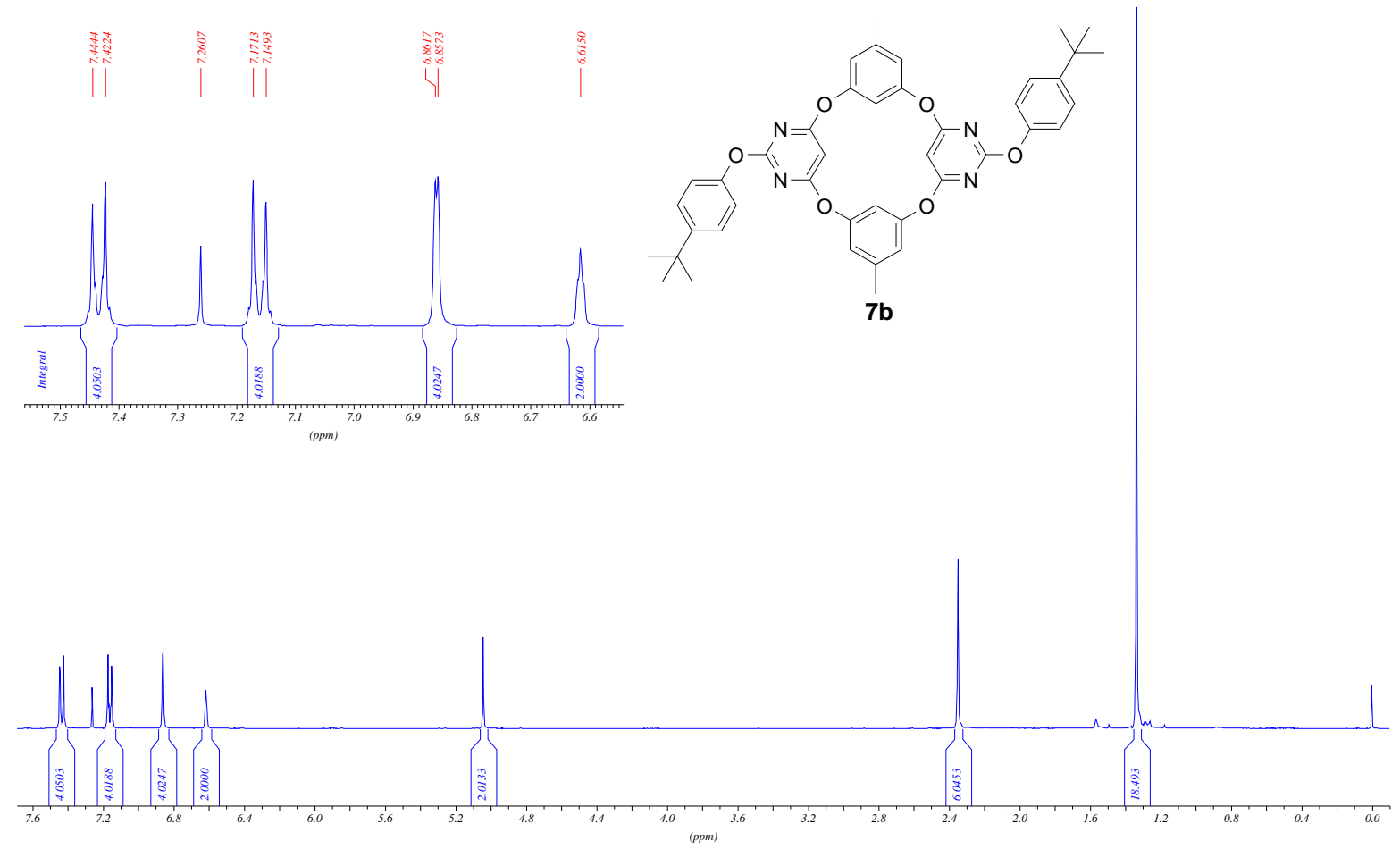

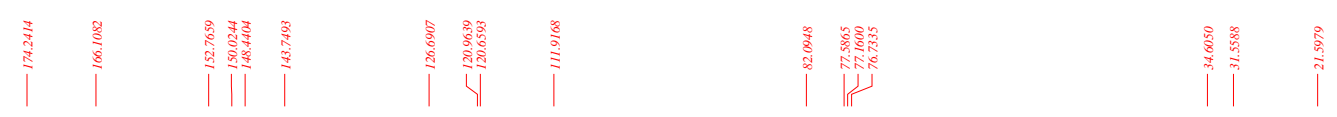

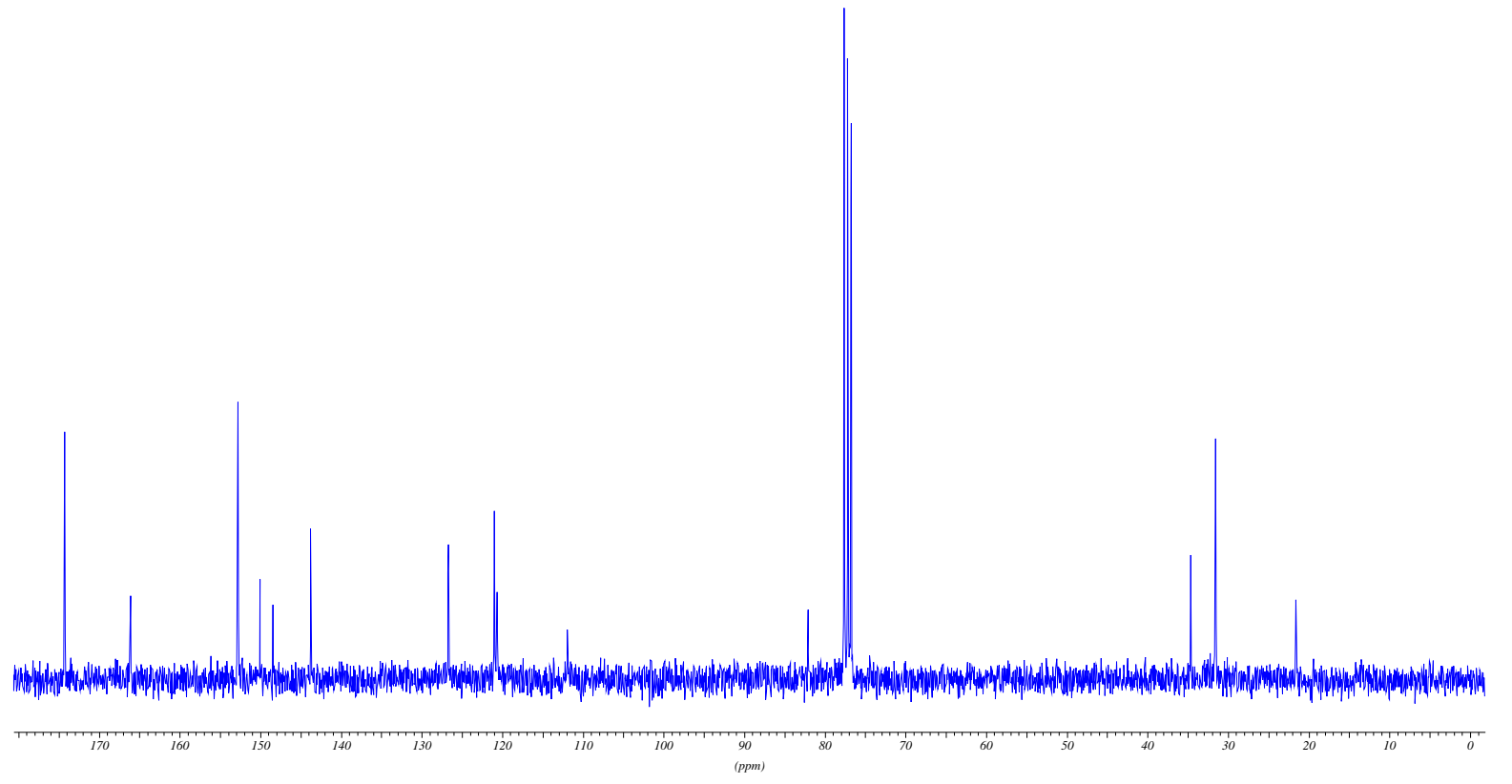




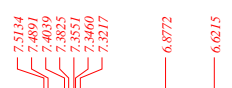
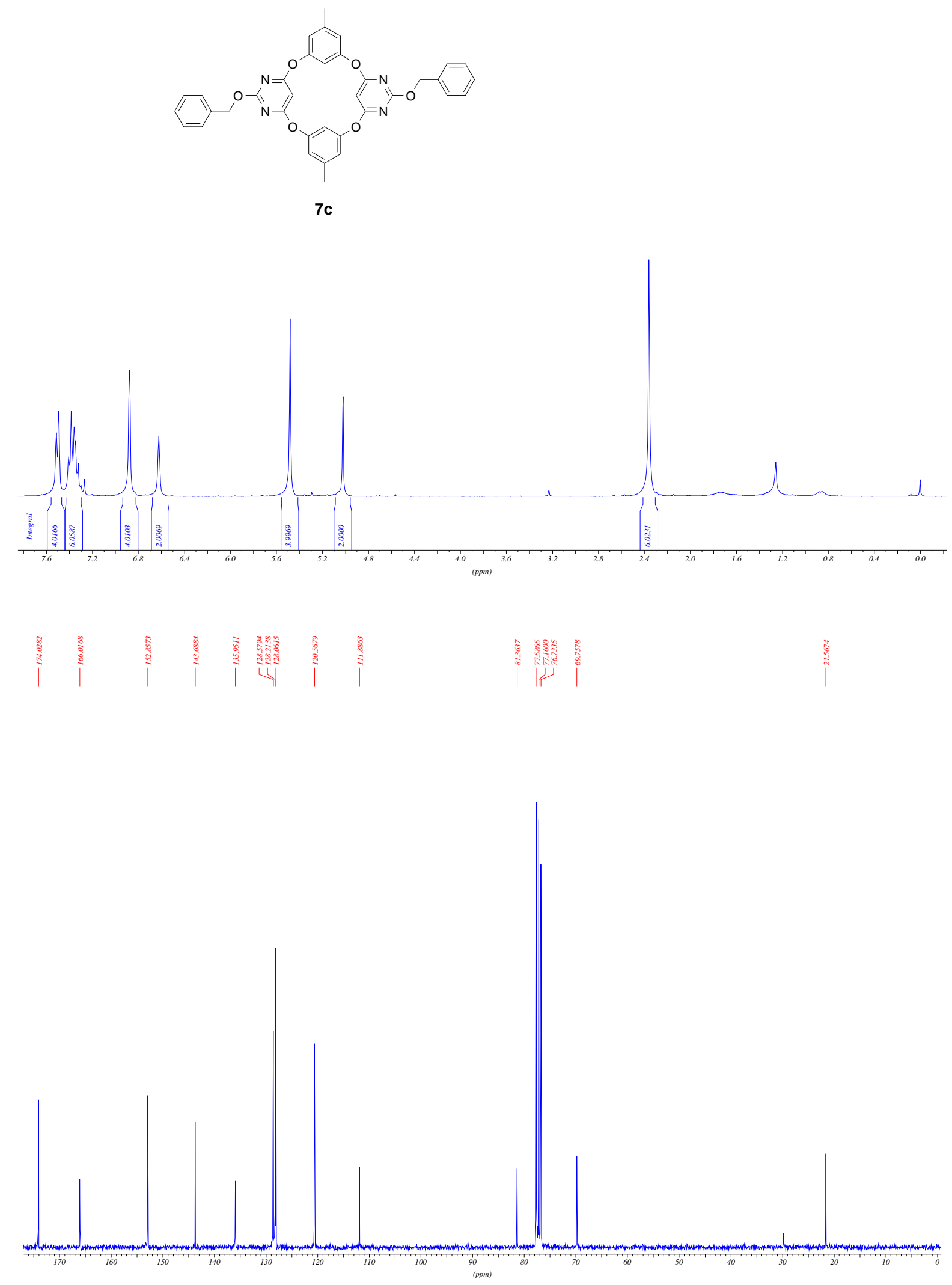

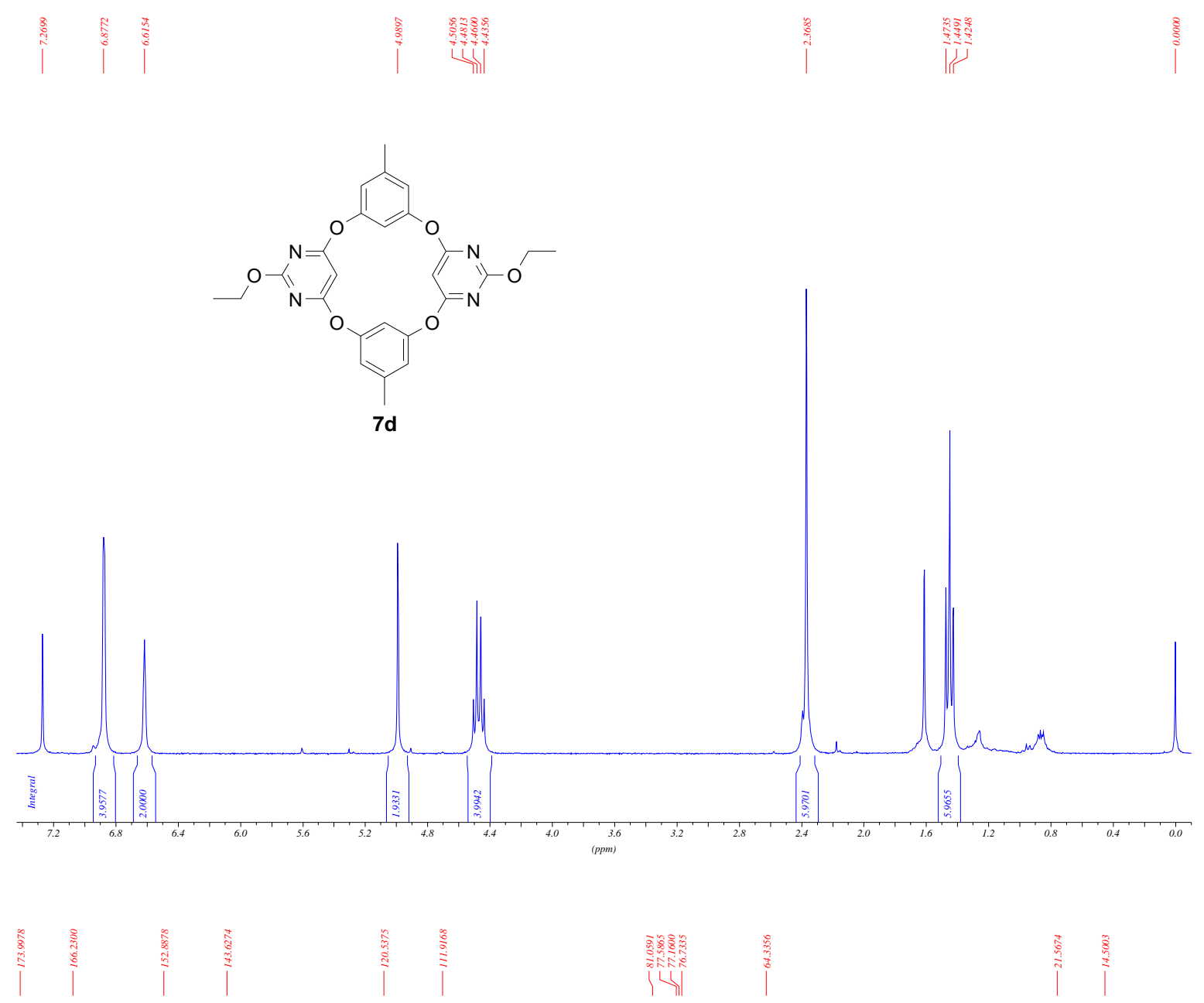

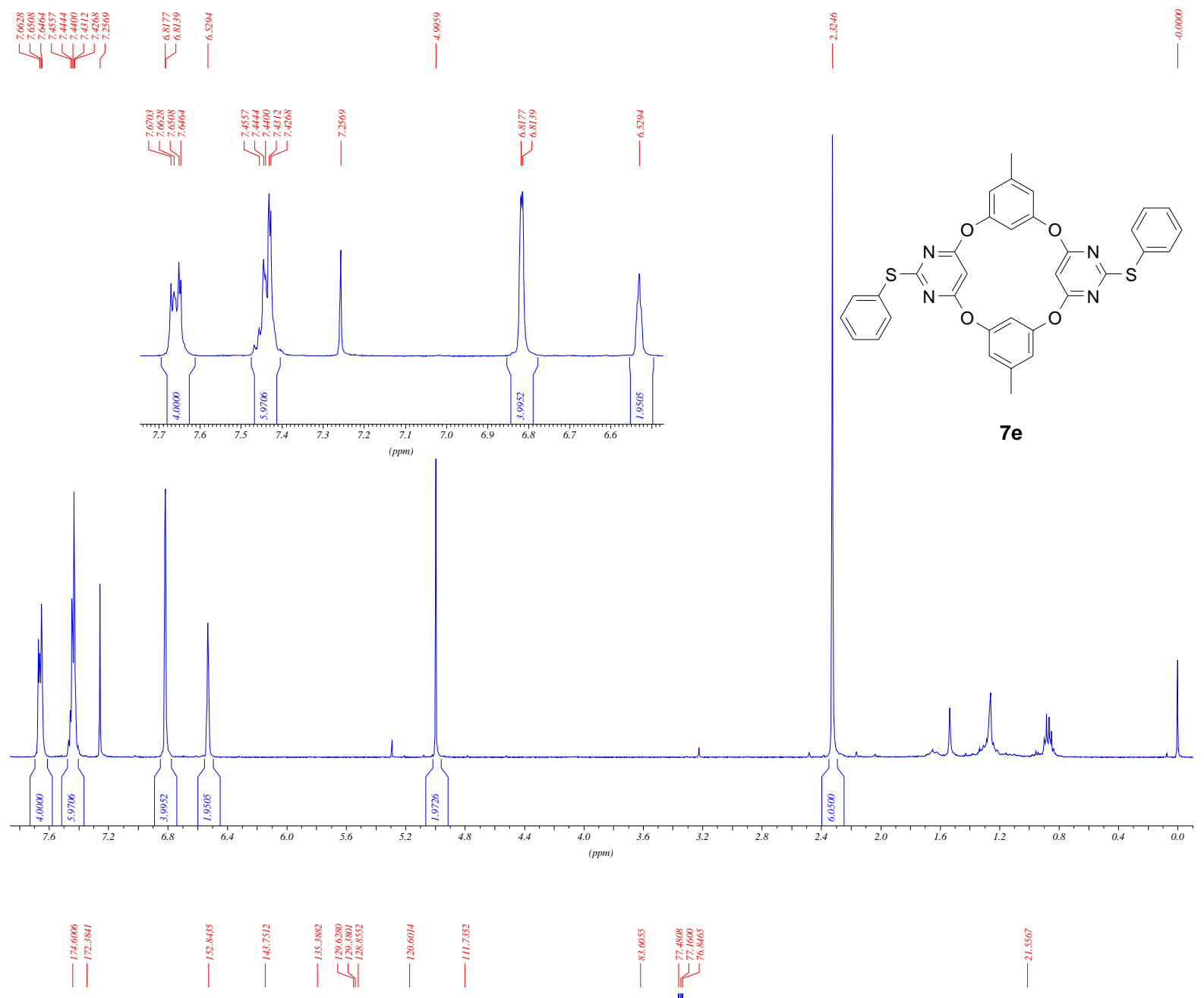

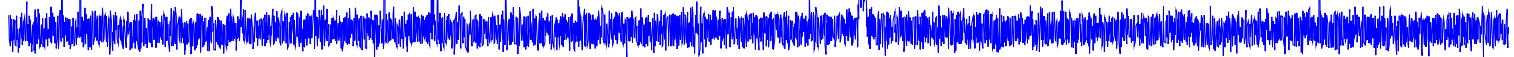



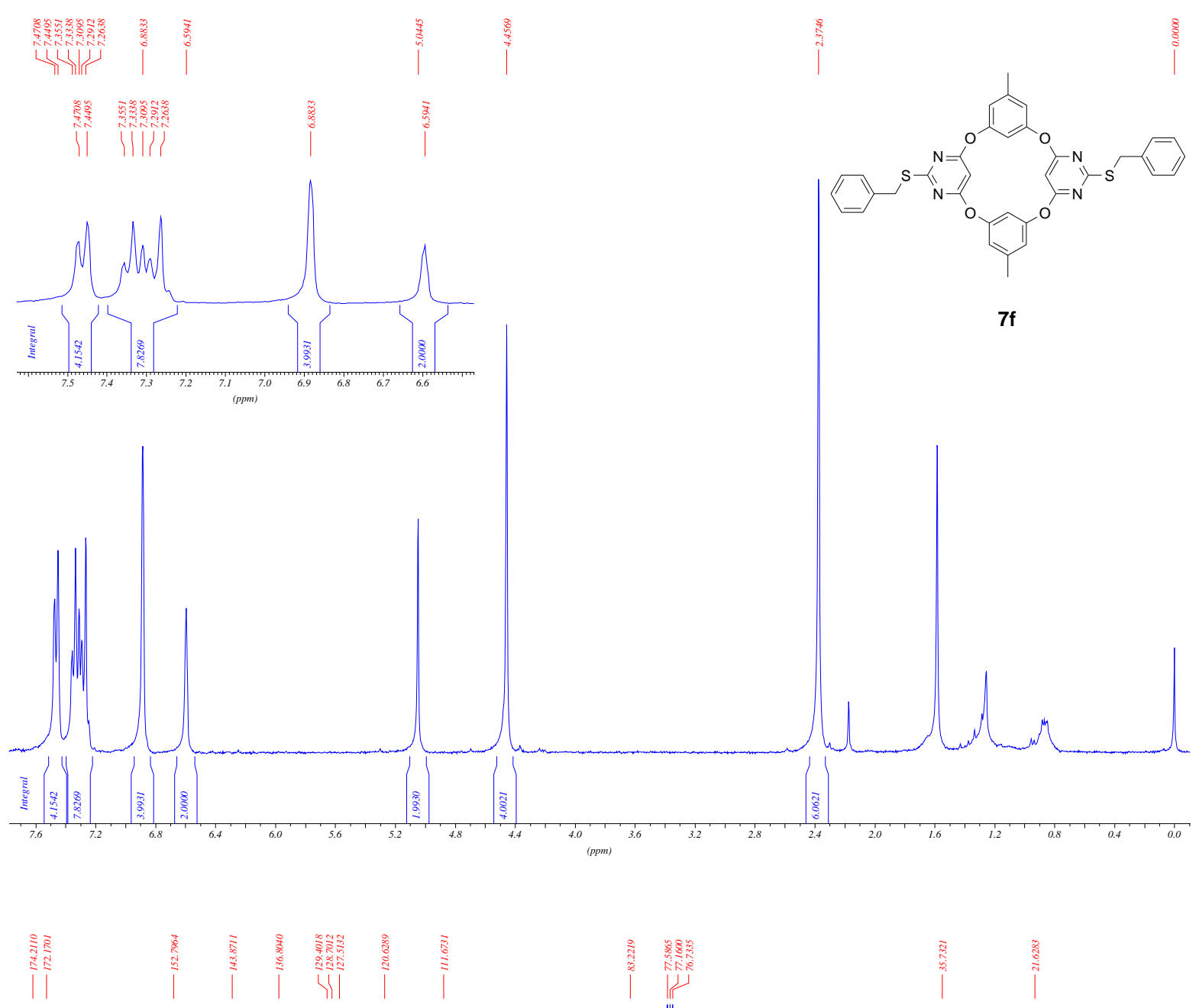


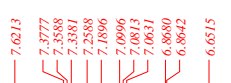

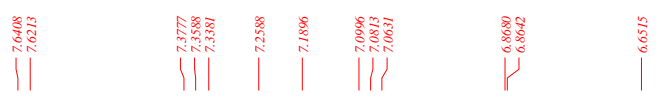
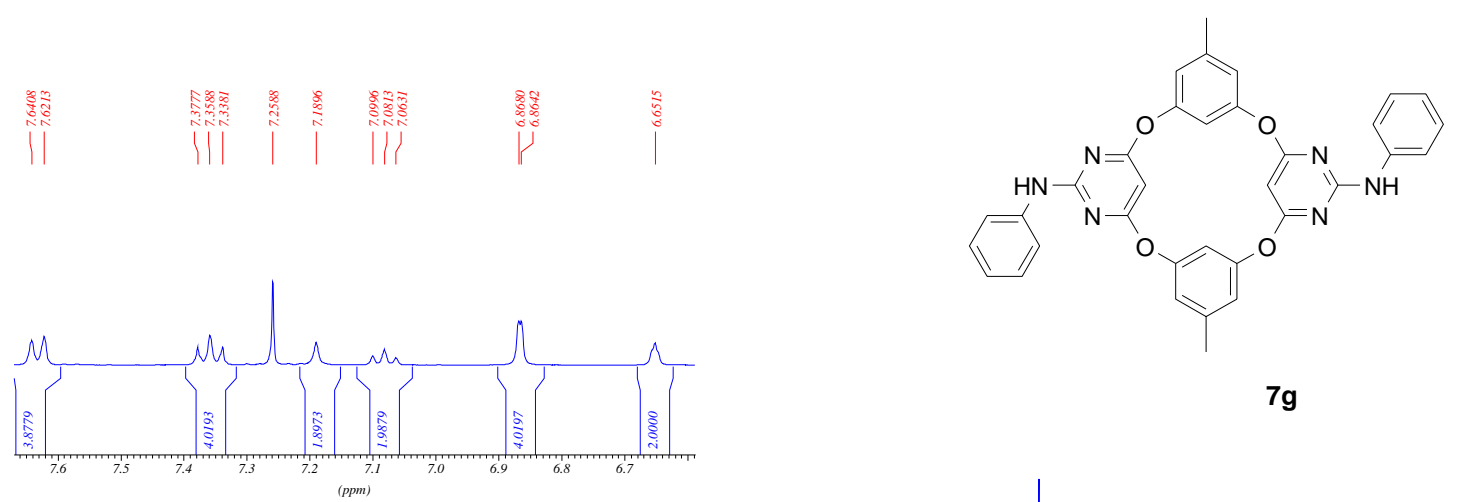

$7 g$

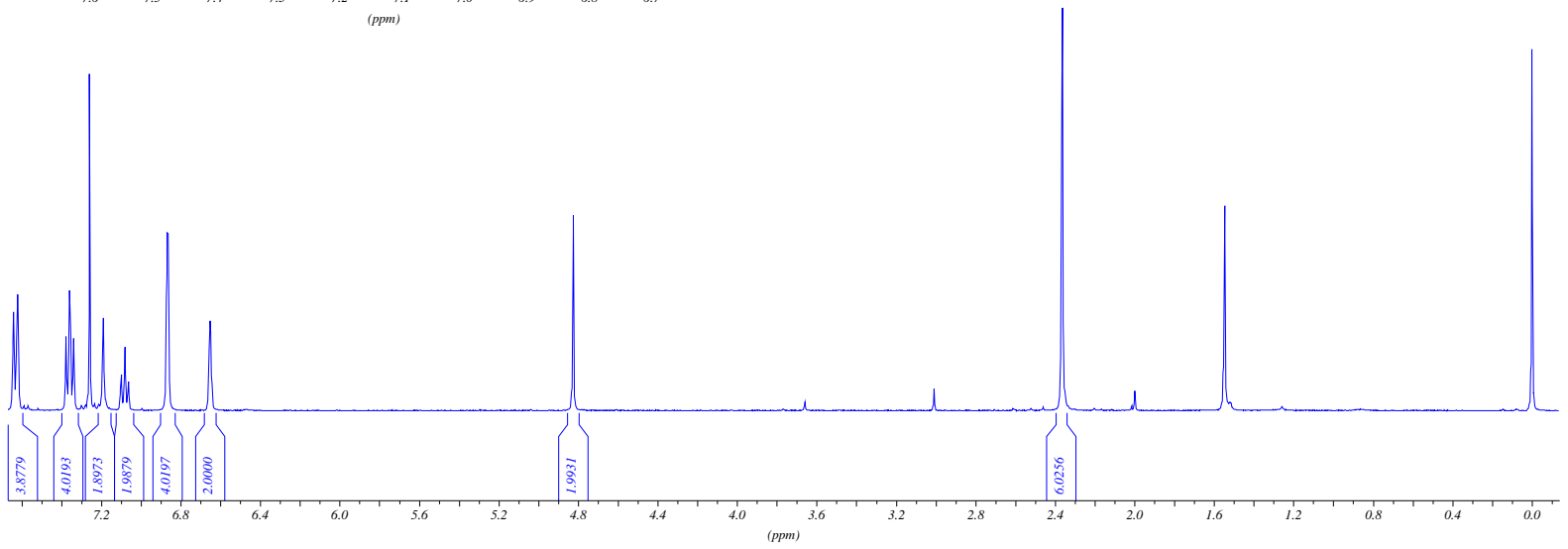

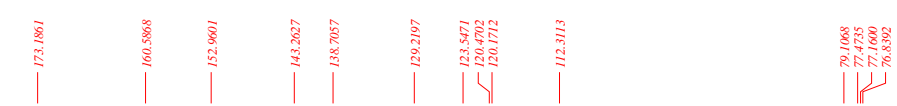
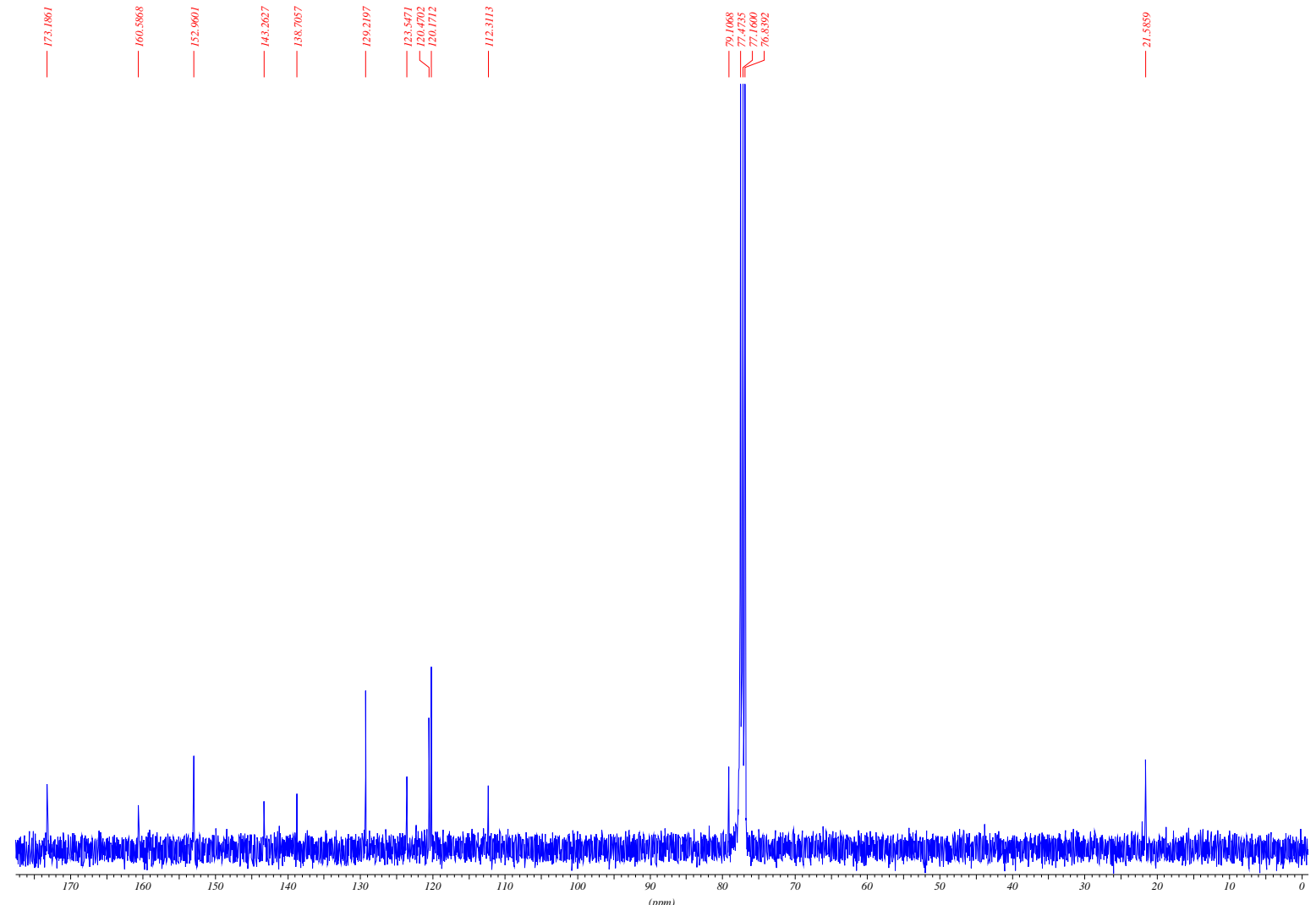


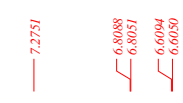
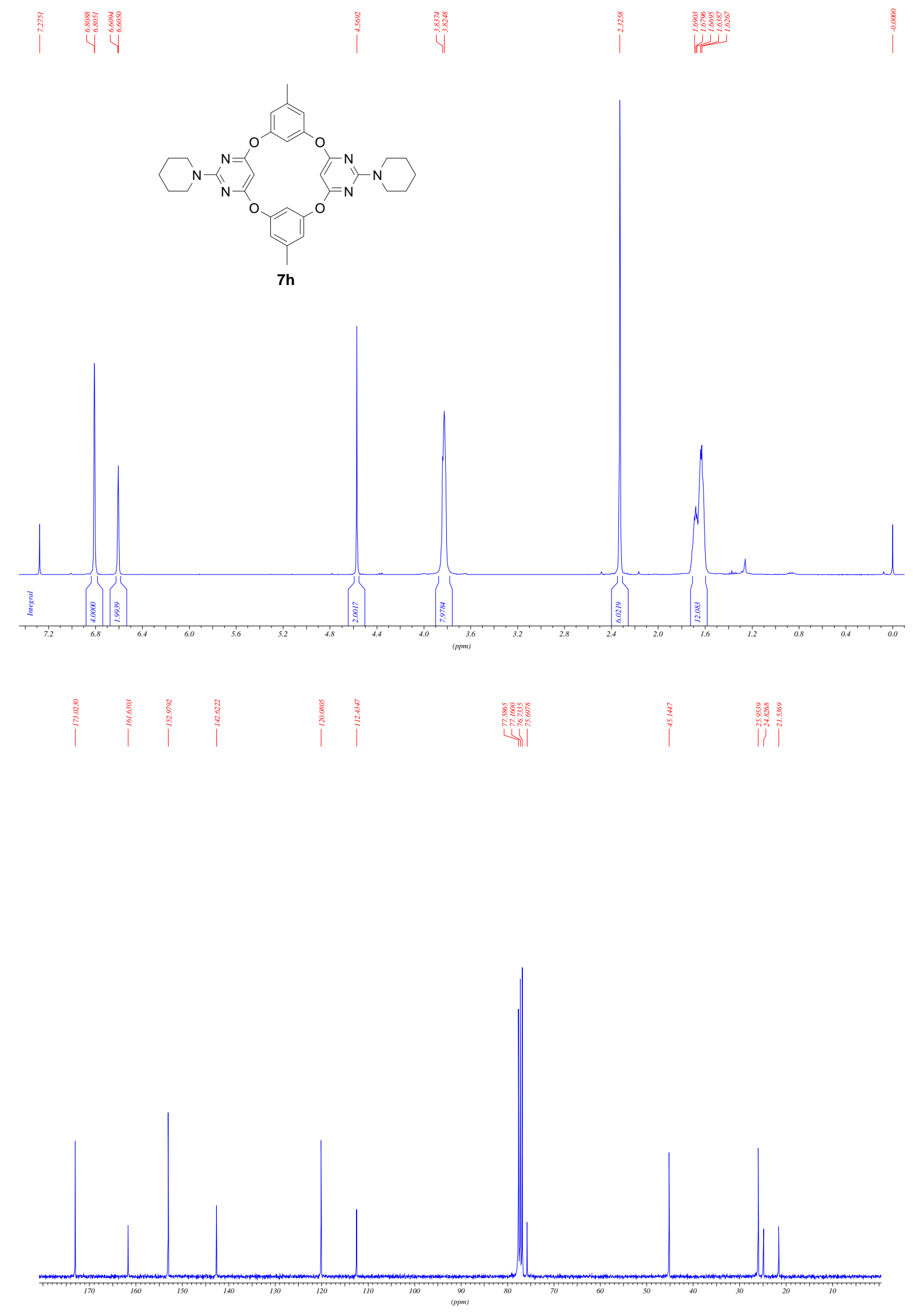

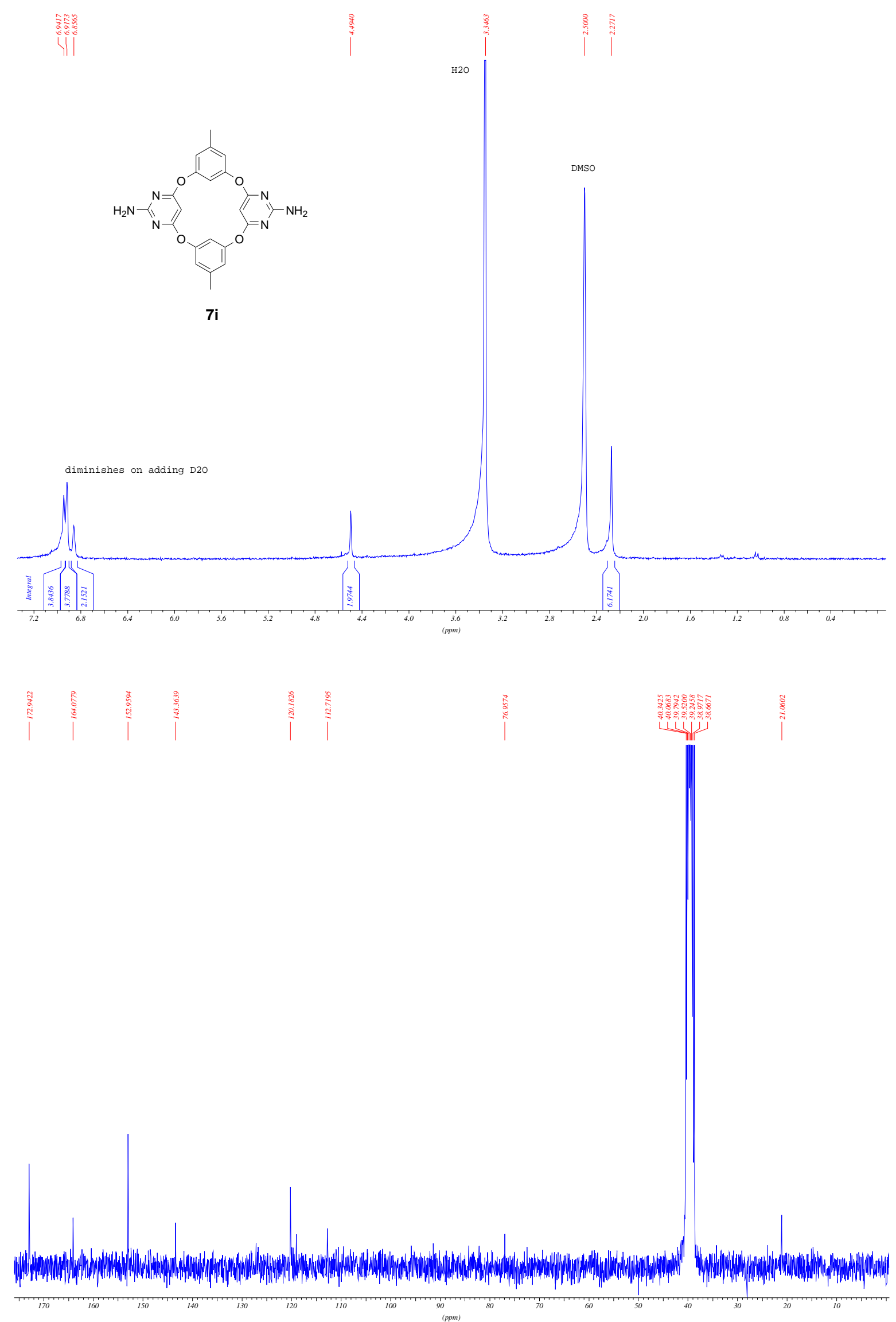

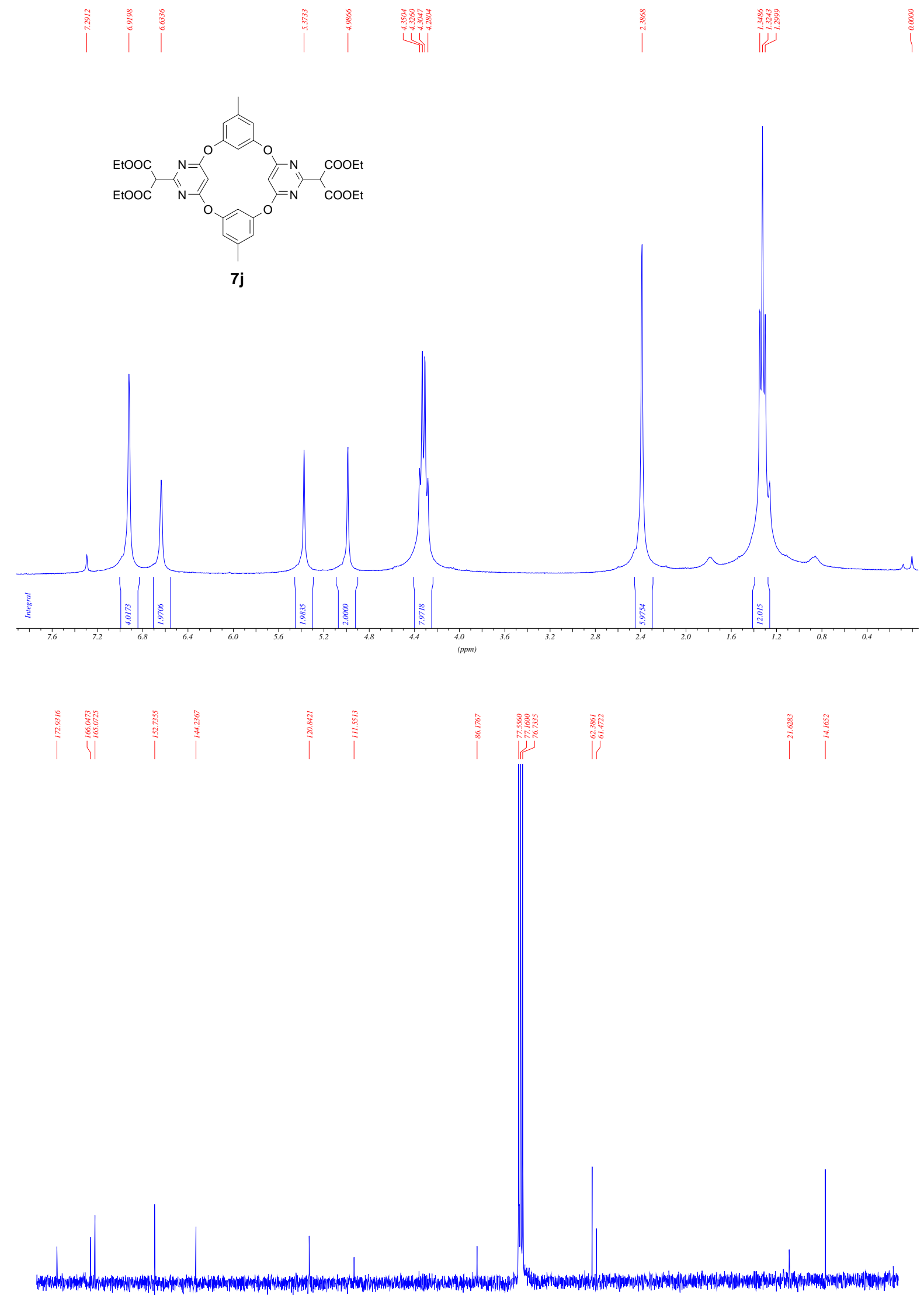


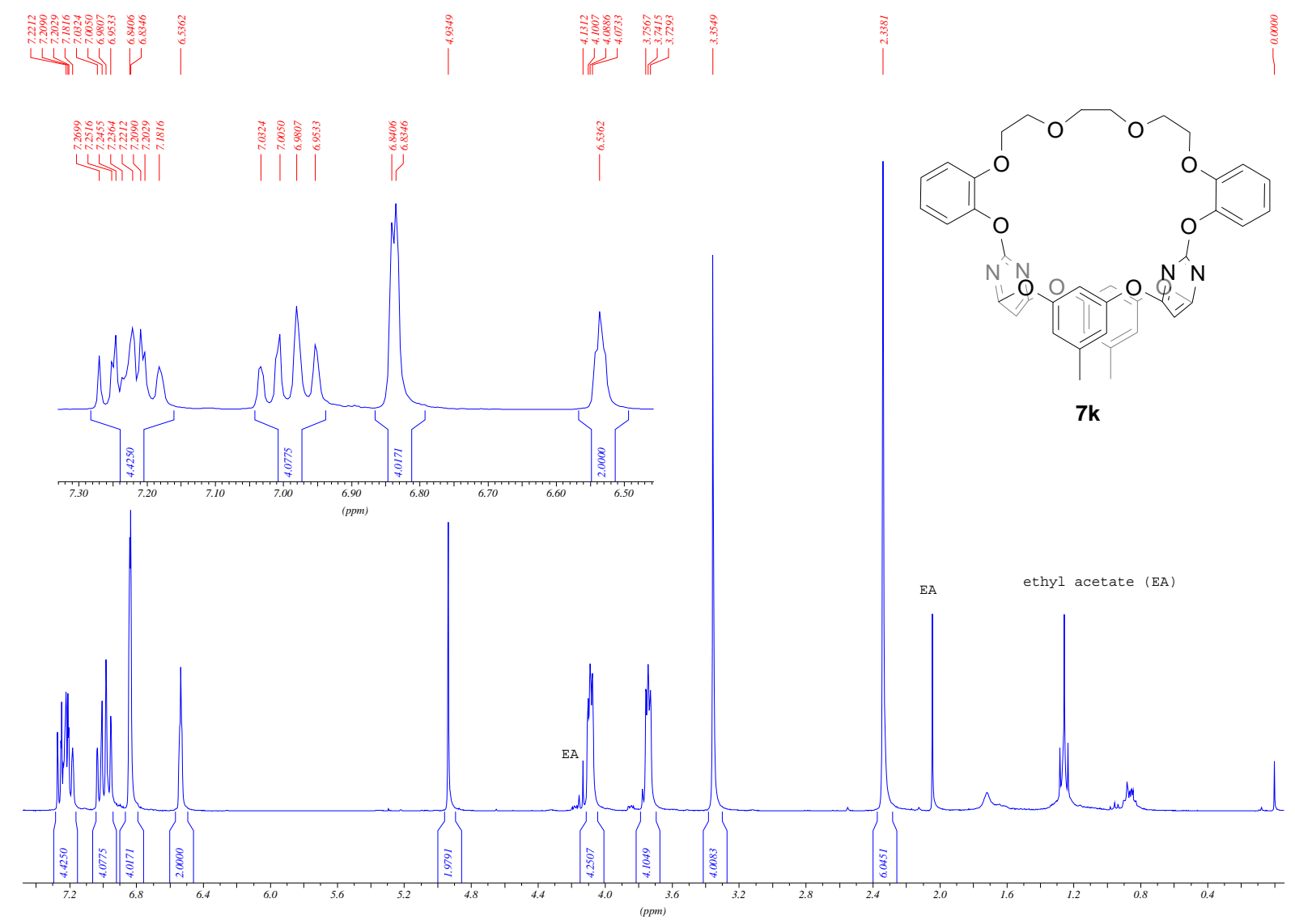

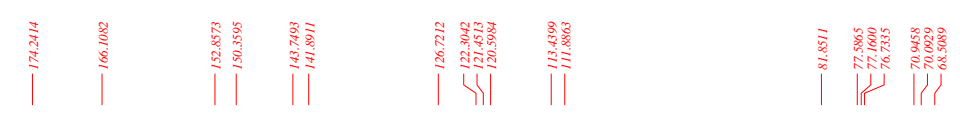

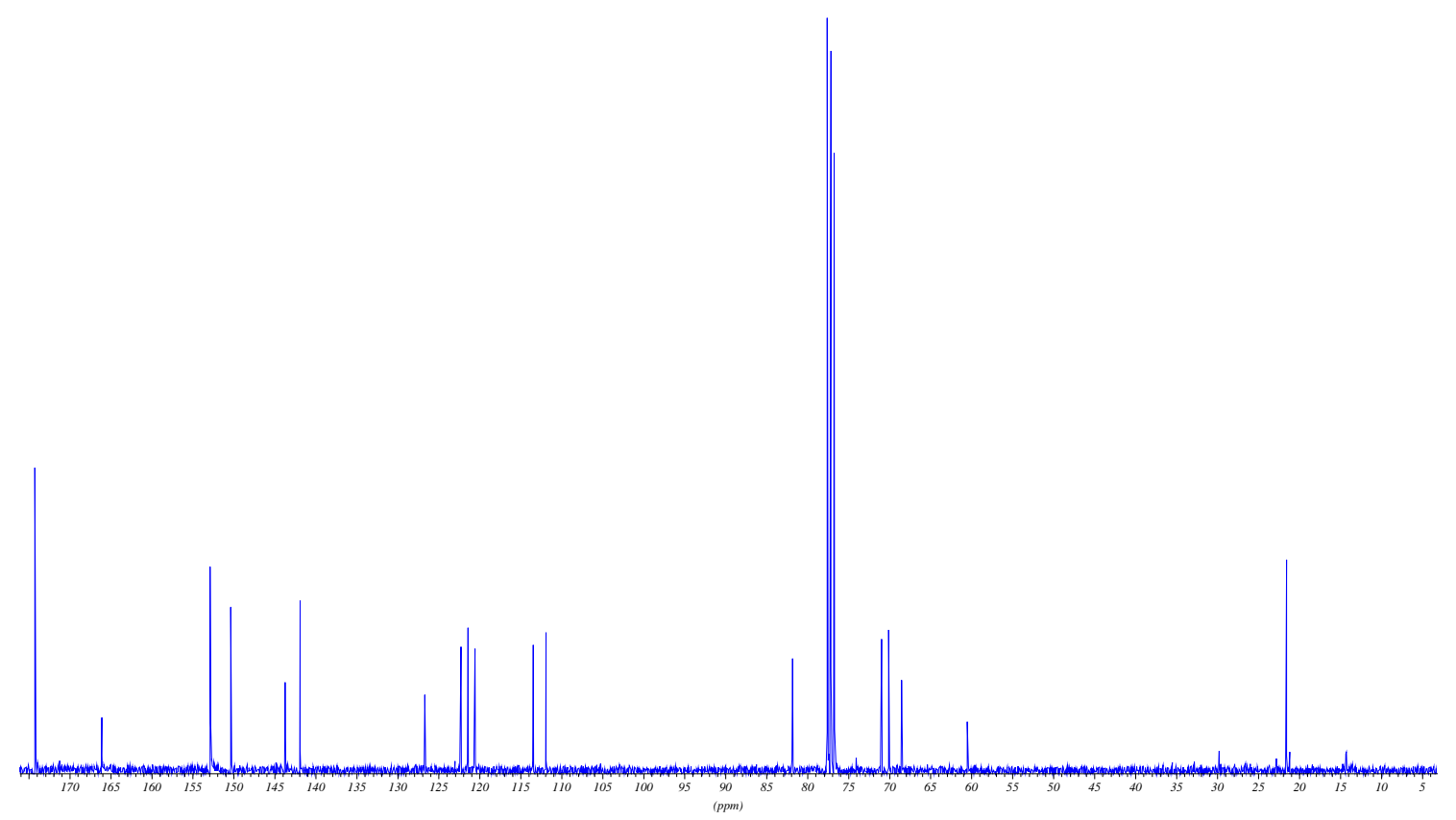



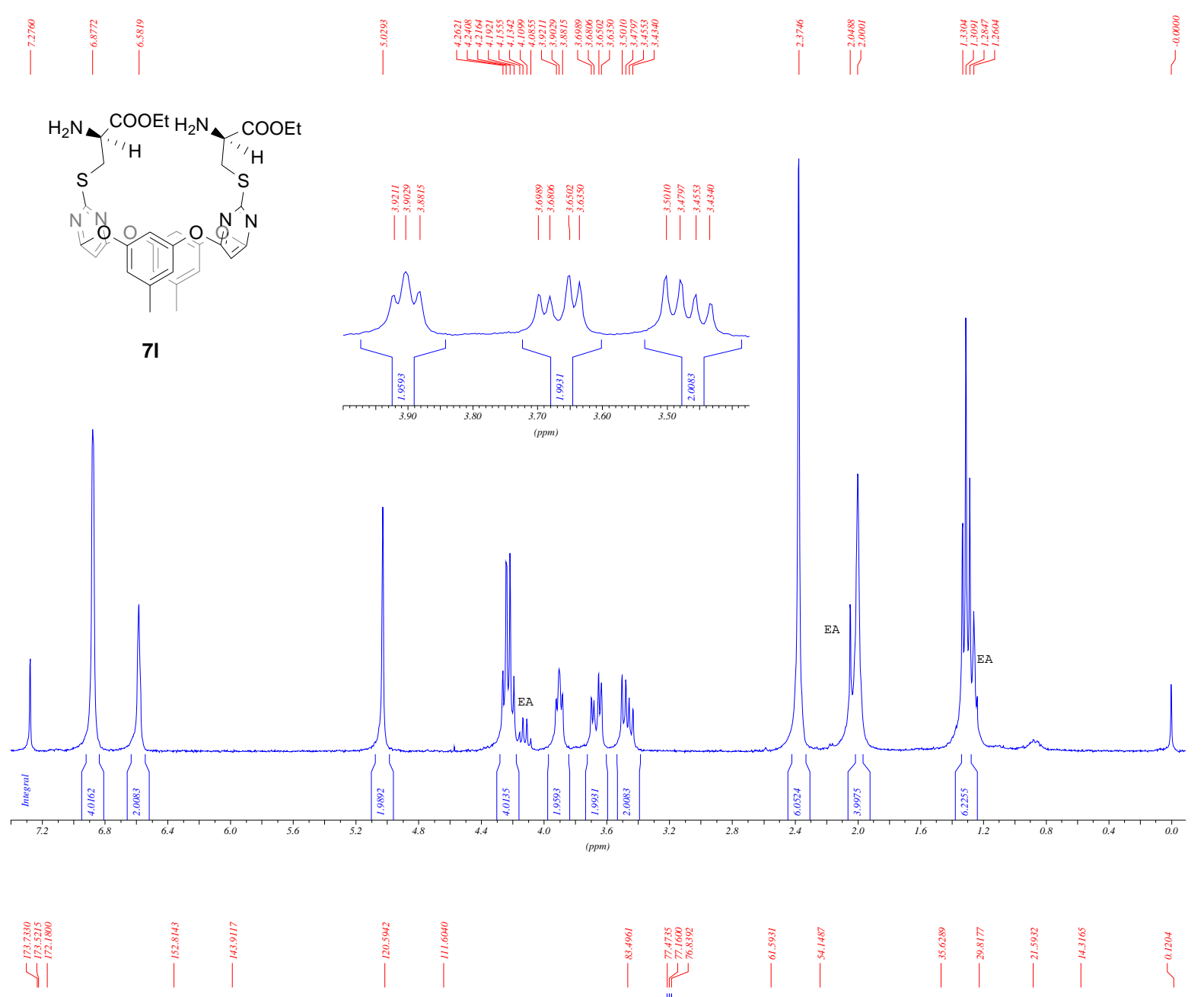

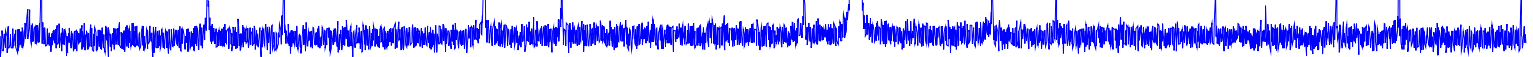

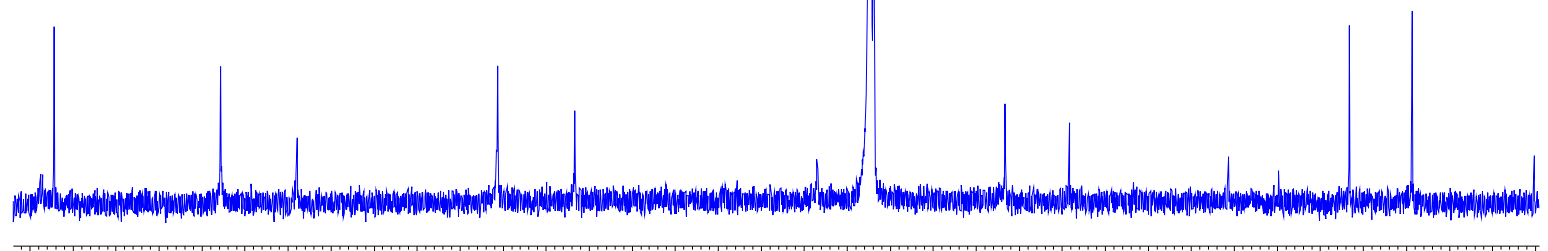
${ }_{(p p m)}$ 


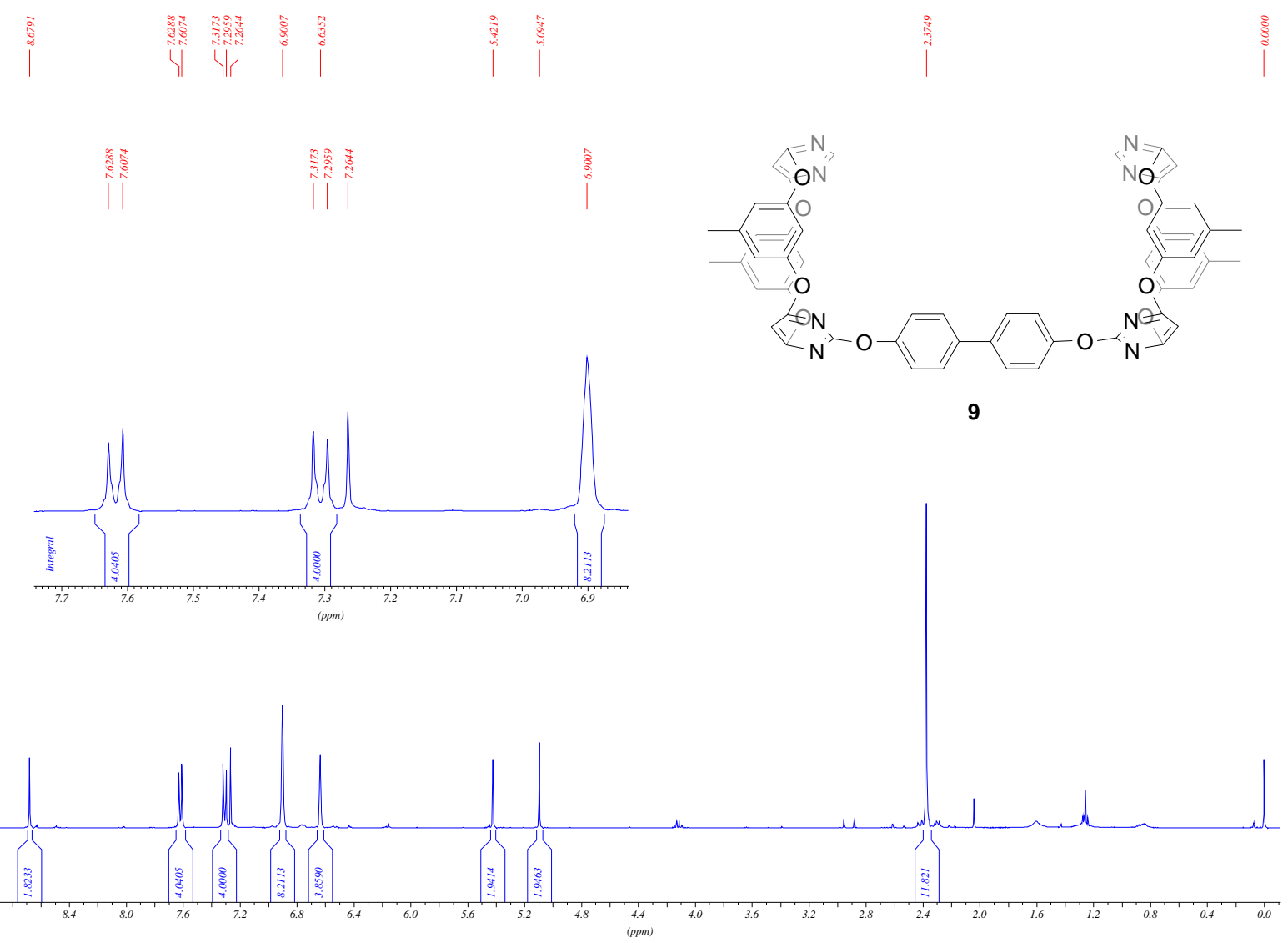

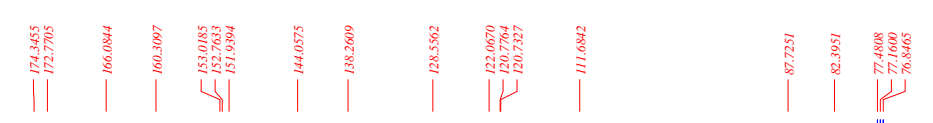




\section{X-ray crystallographic structure and data for oxacalix[2]arene[2]pyrimidine 7k}

The crystals of $\mathbf{7 k}$, grown by vapor diffusion of pentane into a $\mathrm{CHCl}_{3}$ solution of the compound, belong to the monoclinic space group $\mathrm{P} 2{ }_{1} / \mathrm{n}$. A transparent plate with approximate dimensions of $0.3 \mathrm{x}$ $0.2 \times 0.1 \mathrm{~mm}$ was selected for data collection using $\mathrm{CuK} \alpha$ radiation $(\lambda=1.54178 \AA)$ and phi and omega scans. Data were collected at a temperature of $100 \mathrm{~K}$ on a SMART 6000 diffractometer equipped with a CCD detector. Cell refinement and data reduction were carried out by the program $\mathrm{SAINT}^{5}$ on a total of 39363 reflections (8387 independent reflections, $\left.\mathrm{R}_{\text {int }}=13.10\right)$. The structure was solved by direct methods and refined by full-matrix least squares on $\left|\mathrm{F}^{2}\right|$ using the SHELXTL program package ${ }^{6}$ converging to a final $R_{1}=0.0751, \omega R_{2}=0.1895$ for 5118 reflections with $\mathrm{I}_{\mathrm{o}}>2 \sigma\left(\mathrm{I}_{\mathrm{o}}\right)$ and GOOF $=$ 1.075. Non-hydrogen atoms were anisotropically refined and the hydrogen atoms were placed on calculated positions with temperature factors fixed at 1.2 times $U_{\text {eq }}$ of the parent atoms and 1.5 times $\mathrm{U}_{\text {eq }}$ for methyl groups.

* Crystallographic data for the structure of oxacalix[4]arene $7 \mathbf{k}$ :

\begin{tabular}{|c|c|}
\hline formula & $\mathrm{C}_{40} \mathrm{H}_{34} \mathrm{~N}_{4} \mathrm{O}_{10}, 3\left(\mathrm{CHCl}_{3}\right)$ \\
\hline$M\left(\mathrm{gmol}^{-1}\right)$ & $730.2,3 \times 119.4$ \\
\hline crystal dimensions (mm) & $0.3 \times 0.2 \times 0.1$ \\
\hline crystal system & monoclinic \\
\hline space group & $\mathrm{P} 2{ }_{1} / \mathrm{n}$ \\
\hline$a(\AA)$ & $14.6155(12)$ \\
\hline$b(\AA)$ & $22.549(2)$ \\
\hline$c(\AA)$ & $14.7003(12)$ \\
\hline$\alpha(\mathrm{deg})$ & 90.00 \\
\hline$\beta(\operatorname{deg})$ & $100.407(4)$ \\
\hline$\gamma(\operatorname{deg})$ & 90.00 \\
\hline$V\left(\AA^{3}\right)$ & $4764.9(7)$ \\
\hline Z & 4 \\
\hline$\rho_{\text {calc }}\left(\mathrm{gcm}^{3}\right)$ & 1.518 \\
\hline $2 \theta_{\max }(\mathrm{deg})$ & 66.6 \\
\hline wavelength & $\mathrm{CuK} \alpha$ \\
\hline$\lambda(\AA)$ & 1.54178 \\
\hline$F(000)$ & 2223.8 \\
\hline$T(\mathrm{~K})$ & $100(2)$ \\
\hline measured reflections & 39263 \\
\hline unique reflections & 8387 \\
\hline observed reflections $\left(\mathrm{I}_{\mathrm{o}}>2 \sigma\left(\mathrm{I}_{0}\right)\right)$ & 5118 \\
\hline parameters refined & 672 \\
\hline$R_{1}$ & 0.0751 \\
\hline$\omega R_{2}^{\mathrm{a}}$ & 0.1895 \\
\hline$R_{1}$ (all data) & 0.1265 \\
\hline$\omega R_{2}$ (all data) & 0.2216 \\
\hline GOOF & 1.075 \\
\hline$\mu\left(\mathrm{mm}^{-1}\right)$ & 5.354 \\
\hline
\end{tabular}

${ }^{5}$ SAINT, Manual Version 5/6.0, Bruker Analytical X-ray Systems Inc.: Madison, Wisconsin, 1997.

${ }^{6}$ SHELXTL-NT, Manual Version 5.1, Bruker Analytical X-ray Systems Inc.: Madison, Wisconsin, 1997. 
* Angles between the planes through the component aromatic rings:

C2 C3 C4 C5 C6 C7 and C17 C18 C19 C20 C21 C22: 29.53

C25 N26 C27 N28 C29 C30 and C10 N11 C12 N13 C14 C15: $112.32^{\circ}$

* Distances between the bridging O-atoms and the electrophilic (pyrimidine) and nucleophilic (benzene) component aromatic rings:

O-electrophilic ring: average distance: $1.356 \AA$

O-nucleophilic ring: average distance: $1.405 \AA$

Difference $\sim 0.05 \AA$

* Distances between the centroids of the planes through the component aromatic rings:

C2 C3 C4 C5 C6 C7 and C17 C18 C19 C20 C21 C22: $5.430 \AA$

C10 N11 C12 N13 C14 C15 and C25 N26 C27 N28 C29 C30: $6.712 \AA$

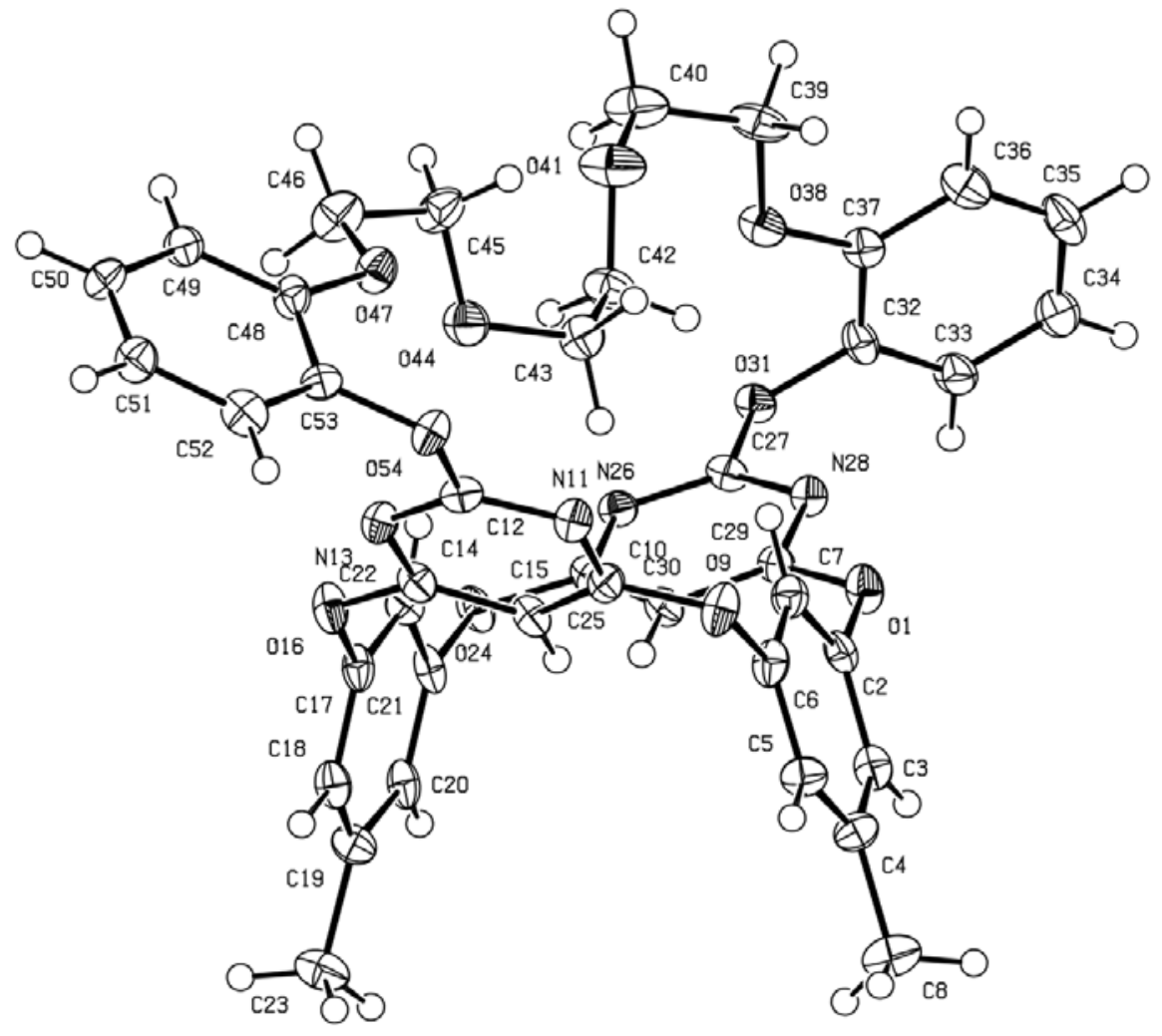

Crystallographic data (excluding structure factors) for the structure reported in this paper have been deposited with the Cambridge Crystallographic Data Centre as supplementary publication no. CCDC 671480. Copies of the data can be obtained free of charge on application to CCDC, 12 Union Road, Cambridge CB2 1EZ, UK (fax: +44(0)-1223-336033 or e-mail: deposit@ccdc.cam.ac.uk). 